DOI 10.4171/JEMS/328

Yucai Su $\cdot$ R. B. Zhang

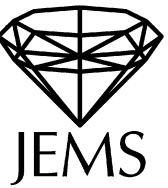

\title{
Generalised Jantzen filtration of Lie superalgebras I
}

Received January 7, 2010, and in revised form September 11, 2010

Abstract. A Jantzen type filtration for generalised Verma modules of Lie superalgebras is introduced. In the case of type I Lie superalgebras, it is shown that the generalised Jantzen filtration for any Kac module is the unique Loewy filtration, and the decomposition numbers of the layers of the filtration are determined by the coefficients of inverse Kazhdan-Lusztig polynomials. Furthermore, the length of the Jantzen filtration for any Kac module is determined explicitly in terms of the degree of atypicality of the highest weight. These results are applied to obtain a detailed description of the submodule lattices of Kac modules.

\section{Contents}

1. Introduction . . . . . . . . . . . . . . . . . . . . . . . . . . 1104

2. Jantzen filtration of Lie superalgebras . . . . . . . . . . . . . . . . . 1106

2.1. Deformed parabolic category $\mathcal{O} \ldots \ldots \ldots \ldots \ldots \ldots$

2.2. Generalised Jantzen filtration ． . . . . . . . . . . . . . . . . . . . . . 1108

2.3. Main problems to be addressed . . . . . . . . . . . . . . . . . . . 1111

3. Jantzen filtration of type I Lie superalgebras . . . . . . . . . . . . . . . . . . 1112

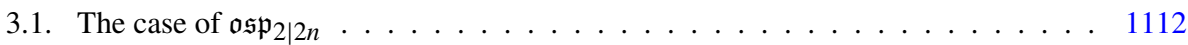

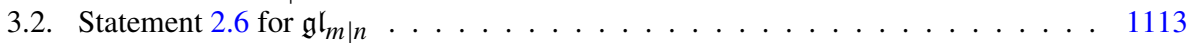

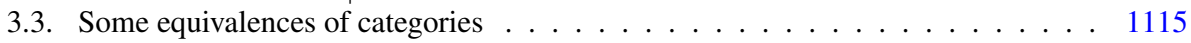

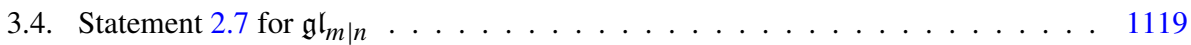

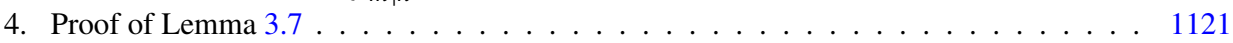

4.1. Primitive weight graphs . . . . . . . . . . . . . . . . . . . . . . . . . . . . . . . . . . . . . . . . 1121

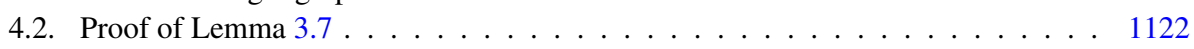

5. Submodule lattices of Kac modules . . . . . . . . . . . . . . . . . . 1123

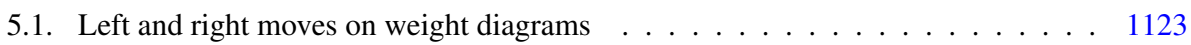

5.2. Technical lemmas . . . . . . . . . . . . . . . . . . . . . 1126

5.3. Primitive weight graphs of Kac modules . . . . . . . . . . . . . . . . . . . . 1130

References . . . . . . . . . . . . . . . . . . . . . . 1131

Y. Su: Department of Mathematics, Tongji University, Shanghai 200092, China, and Wu Wen-Tsun Key Laboratory of Mathematics, USTC, Hefei 230026, China; e-mail: ycsu @tongji.edu.cn R. B. Zhang: School of Mathematics and Statistics, University of Sydney, Sydney, Australia; e-mail: rzhang@maths.usyd.edu.au 


\section{Introduction}

In the late 1970s, Jantzen introduced a filtration [22, 23] for Verma modules over semisimple complex Lie algebras, which now bears his name. He also formulated precise conjectures on the Jantzen filtration, which turned out to be closely related to (in fact [16], imply) the Kazhdan-Lusztig conjecture [25]. The Kazhdan-Lusztig and Jantzen conjectures were foci of representation theory in the 1980s. The Kazhdan-Lusztig conjecture was proven by Beilinson and Bernstein [3] and by Brylinski and Kashiwara [10] independently, and the Jantzen conjectures were also settled in the affirmative by Beilinson and Bernstein [4]. These developments are among the most important achievements in representation theory in recent times.

The Jantzen conjectures are as deep as the Kazhdan-Lusztig conjecture (this was already indicated in the early work [16]). Their proof required far-reaching generalisations of the geometric techniques used in the proof of the Kazhdan-Lusztig conjecture. In very brief terms, the essential idea of the proof in [4] is to enrich the relevant category of perverse sheaves with extra structures. This then allows one to interpret the Jantzen filtration as a weight filtration in the sense of Gabber on the side of perverse sheaves.

The Jantzen filtration has been generalised to other contexts [1, 2] by Andersen. There is also a close relationship between the Jantzen filtration for Verma modules and Koszul grading in the context of category $\mathcal{O}$ developed in the influential paper [5] of Beilinson, Ginzburg and Soergel. For recent developments along these lines, we refer to [30, 31] and references therein.

In the present paper and its sequel, we introduce a Jantzen type filtration for generalised Verma modules of classical Lie superalgebras [24, 27] over $\mathbb{C}$ and study its properties. For each such Lie superalgebra, we take the upper triangular maximal parabolic subalgebra with a purely even Levi subalgebra. Then the generalised Verma modules under study are those induced by finite-dimensional irreducible modules over the maximal parabolic subalgebra, and all generalised Verma modules in this paper will be assumed to be of this kind. For type I Lie superalgebras (that is, $\mathfrak{o s p}_{2 \mid 2 n}$ and the general and special linear superalgebras), such generalised Verma modules are finite-dimensional and are usually referred to as Kac modules. However, for type II Lie sueparlgebras, such generalised Verma modules are always infinite-dimensional.

The Jantzen type filtration for generalised Verma modules of Lie superalgebras is defined in essentially the same way as the original Jantzen filtration of ordinary Lie algebras. In particular, we shall closely follow $[16,30]$ in working over the power series $\operatorname{ring} T:=$ $\mathbb{C}[[t]]$ in the variable $t$. We first construct a filtration for each generalised Verma module over $T$ by using a natural non-degenerate contravariant $T$-bilinear form defined on it. Then by specialising to the field of complex numbers, we obtain the generalised Jantzen filtration for the corresponding generalised Verma module over $\mathbb{C}$. Details of the construction are given in Section 2.2. We also introduce polynomials in one variable with the coefficients being decomposition numbers of the consecutive quotients of a generalised Jantzen filtration (see (2.14)). For easy reference, we call them Jantzen polynomials.

One of the main questions to be addressed is whether the consecutive quotients of a generalised Jantzen filtration are semisimple. If the answer is affirmative, we shall also 
determine whether it is a Loewy filtration. The other main question to be addressed is whether the Jantzen polynomials are equal to the inverse Kazhdan-Lusztig polynomials [28] of the Lie superalgebra defined in terms of Kostant cohomology. More precise descriptions of the questions are given in Statement 2.6 and Statement 2.7.

Investigations into the above questions were motivated by the Jantzen conjectures, and also prompted by the examples $\mathfrak{g l}_{m \mid n}$ for $n=1,2$, for which both questions have affirmative answers. This is quite trivial to see for all Kac modules over $\mathfrak{g l}_{m \mid 1}$ and for the typical and singly atypical Kac modules over $\mathfrak{g l}_{m \mid 2}$. The other Kac modules over $\mathfrak{g l}_{m \mid 2}$ have the same structure as the doubly atypical Kac modules over $\mathfrak{g l}_{2 \mid 2}$ by a result of Serganova [29, Theorem 2.6]. For the latter algebra, the Jantzen filtration for every Kac module can be worked out explicitly, and the inverse Kazhdan-Lusztig polynomials were also known [39]. Inspecting the results one sees that the above questions have affirmative answers in this case as well.

Here we shall deal with the Jantzen filtration of type I Lie superalgebras; type II Lie superalgebras will be treated in the sequel to this paper.

We show that for $\mathfrak{g}$ being a type I Lie superalgebra, the Jantzen filtration for any Kac module over $\mathfrak{g}$ is a Loewy filtration. The length of the Jantzen filtration is also determined explicitly in terms of the degree of atypicality of the highest weight (see the beginning of Subsection 2.1 for the terminology). In the case $\mathfrak{g}=\mathfrak{o} \mathfrak{s p}_{2 \mid 2 n}$, a Kac module can have at most two composition factors. From this one can easily deduce that Kac modules for $\mathfrak{o s p}_{2 \mid 2 n}$ are rigid. By a result of Brundan and Stroppel [9, IV], Kac modules for $\mathfrak{g}=\mathfrak{g l}_{m \mid n}$ (and thus also $\mathfrak{s l}_{m \mid n}$ ) are rigid. Therefore, the Jantzen filtration for a Kac module over $\mathfrak{g}$ is the unique Loewy filtration, which necessarily coincides with the socle filtration and radical filtration. These results are summarised in Theorems 3.2, 3.3 and 3.6.

We also show that for type I Lie superalgebras, the Jantzen polynomials coincide with the inverse Kazhdan-Lusztig polynomials (see Theorem 3.3 for $\mathfrak{o s p}_{2 \mid 2 n}$ and Theorem 3.16 for $\left.\mathfrak{g l}_{m \mid n}\right)$. In the case of $\mathfrak{o s p}_{2 \mid 2 n}$, a formula for the Kazhdan-Lusztig polynomials was given in [40, Corollary 6.4], from which one can easily deduce a formula for the inverse Kazhdan-Lusztig polynomials. The Jantzen polynomials are also easy to write down in explicit form. Inspecting the results, we immediately see that the Jantzen polynomials are equal to the inverse Kazhdan-Lusztig polynomials. In the type $A$ case, we make use of the "super duality" conjectured in [13] and proved very recently in [11], [9, IV] to transcribe the problem to ordinary general linear algebra. Then we deduce Theorem 3.16 from results obtained by Collingwood, Irving and Shelton [15], Boe and Collingwood [6] and Irving [21] on Loewy filtrations of generalised Verma modules for the general linear algebra.

We also study the submodule lattices of Kac modules. By using results on the Jantzen filtration combined with combinatorics of weight diagrams [9, 17], we obtain a detailed description of the chains in the submodule lattices of Kac modules. In particular, a necessary and sufficient condition is given for one indecomposable submodule to cover another in the submodule lattice of a Kac module in Theorem 5.18. We should point out that Hughes, King and van der Jeugt conjectured an array of structural properties of Kac modules twenty years ago [19], but did not publish their findings. Their conjectures were based on extensive computations and included the statement of Theorem 5.18. 
Let us briefly comment on the Jantzen filtration of type II Lie superalgebras. In the case of $\mathfrak{o s p} p_{m \mid 2}$, one can deduce from results on the structure of the generalised Kac modules which were studied in [37] that consecutive quotients of their Jantzen filtrations are semisimple. In fact both Statements 2.6 and 2.7 hold for $\mathfrak{o s p}_{m \mid 2}$. As the techniques required for studying the Jantzen filtration of the type II Lie superalgebras are quite different from those used in this paper, we shall present the full treatment of type II Lie superalgebras in a separate publication.

The organisation of the paper is as follows. In Section 2, we introduce the generalised Jantzen filtration for a class of generalised Verma modules of Lie superalgebras, and state the main problems (whether Statements 2.6 and 2.7 are true) to be addressed in this paper and its sequel. In Section 3, we show that both Statements 2.6 and 2.7 are true for type I Lie superalgebras, thus gaining a thorough understanding of the layers of the Jantzen filtration for Kac modules. A technical result (Lemma 3.7) used in Section 3 is proved in Section 4. Finally in Section 5, we apply the results of Section 3 to study the submodule lattices of Kac modules. The main result obtained is Theorem 5.18.

\section{Jantzen filtration of Lie superalgebras}

\subsection{Deformed parabolic category $\mathcal{O}$}

Given any complex Lie superalgebra $\mathfrak{a}$, we let $\mathfrak{a}_{\overline{0}}$ and $\mathfrak{a}_{\overline{1}}$ be the even and odd subspaces respectively, and denote by $\mathrm{U}(\mathfrak{a})$ its universal enveloping algebra over the complex number field $\mathbb{C}$. Let $\mathfrak{g}=\mathfrak{g}_{0} \oplus \mathfrak{g}_{1}$ be either the complex general linear superalgebra $\mathfrak{g l}_{m \mid n}$, or a finite-dimensional classical simple Lie superalgebra $[24,27]$ over $\mathbb{C}$. Let $\mathfrak{h} \subset \mathfrak{g}_{0}^{-}$be a Cartan subalgebra of $\mathfrak{g}$, and choose the distinguished Borel subalgebra $\mathfrak{b}$ containing $\mathfrak{h}$ such that the corresponding set $\Pi$ of simple roots contains a unique odd simple root $\alpha_{s}$ [24, Table VI] (see also [27]). Denote by $\Delta_{\overline{0}}^{+}$and $\Delta_{\overline{1}}^{+}$respectively the sets of even and odd positive roots with respect to $\mathfrak{b}$. Let $\rho_{0}=\frac{1}{2} \sum_{\alpha \in \Delta_{\overline{0}}} \alpha, \rho_{1}=\frac{1}{2} \sum_{\gamma \in \Delta_{\overline{1}}} \gamma$ and $\rho=\rho_{0}-\rho_{1}$. Set $\Delta^{+}=\Delta_{\overline{0}}^{+} \cup \Delta_{\overline{1}}^{+}$and $\Delta=-\Delta^{+} \cup \Delta^{+}$.

We denote $\Delta_{1}^{+}=\left\{\gamma \in \Delta_{\overline{1}}^{+} \mid 2 \gamma \notin \Delta_{\overline{0}}^{+}\right\}$. Given $\mu \in \mathfrak{h}^{*}$, if there exists $\gamma \in \Delta_{1}^{+}$such that $(\mu+\rho, \gamma)=0$, we say that $\mu$ is atypical and $\gamma$ is an atypical root of $\mu$. The degree $\sharp(\mu)$ of atypicality of $\mu$ is the maximal number of its mutually orthogonal atypical roots in $\Delta_{1}^{+}$. If $\sharp(\mu)=0$, we say that the weight $\mu$ is typical.

For each root $\alpha \in \Delta$, we denote by $\mathfrak{g}_{\alpha}$ the root space associated to it. Let $\mathfrak{p}$ be the parabolic subalgebra of $\mathfrak{g}$ generated by $\mathfrak{h}, \mathfrak{g}_{\alpha_{s}}$ and all $\mathfrak{g}_{ \pm \alpha}$ with $\alpha_{s} \neq \alpha \in \Pi$. Then $\mathfrak{p}=\mathfrak{l}+\mathfrak{u}$ with $\mathfrak{l}$ being the Levi subalgebra and $\mathfrak{u}$ the nilradical. We denote by $\Delta(\mathfrak{u})$ the roots of $\mathfrak{u}$. Let $\overline{\mathfrak{u}}$ be the nilpotent subalgebra of $\mathfrak{g}$ spanned by the root spaces $\mathfrak{g}_{-\beta}$ with $\beta \in \Delta(\mathfrak{u})$. Then $\mathfrak{g}=\overline{\mathfrak{u}}+\mathfrak{l}+\mathfrak{u}$. Note that $\mathfrak{l} \subset \mathfrak{g}_{0}$ is a reductive Lie algebra and is purely even. We denote by $\Delta^{+}(\mathfrak{l}) \subset \Delta_{\overline{0}}^{+}$the set of positive roots of $\mathfrak{l}$. Then every $\alpha \in \Delta^{+}(\mathfrak{l})$ satisfies $(\alpha, \alpha) \neq 0$. Let

$$
P_{0}^{+}=\left\{\mu \in \mathfrak{h}^{*} \mid \frac{2(\mu, \alpha)}{(\alpha, \alpha)} \in \mathbb{Z}_{+}, \forall \alpha \in \Delta^{+}(\mathfrak{l})\right\}
$$

be the set of integral $\mathfrak{l}$-dominant weights. 
Let $T:=\mathbb{C}[[t]]$ be the ring of formal power series in the indeterminate $t$, and consider $\mathbb{C}$-algebra homomorphisms $\phi: \mathrm{U}(\mathfrak{h}) \rightarrow T$ of the following kind. For $\alpha \in \Delta^{+}(\mathfrak{l})$, denote by $\check{\alpha}$ the coroot (i.e., $\check{\alpha} \in \mathfrak{h}$ satisfying $\mu(\check{\alpha})=2(\mu, \alpha) /(\alpha, \alpha)$ for all $\left.\mu \in \mathfrak{h}^{*}\right)$. We require $\phi$ to satisfy the following conditions:

$$
\phi(\check{\alpha})=0 \quad \text { for all } \alpha \in \Delta^{+}(\mathfrak{l}), \quad \operatorname{Im} \phi \subset t \mathbb{C}[[t]] \quad \text { and } \quad \frac{\operatorname{Im} \phi}{t^{2} \mathbb{C}[[t]]} \cong \mathbb{C} .
$$

Such morphisms exist in abundance. For example, we may take

$$
\begin{aligned}
& \phi(h)=t \delta(h) \quad \text { for all } h \in \mathfrak{h}, \text { with fixed } \delta \in \mathfrak{h}^{*} \text { satisfying } \\
& (\delta, \alpha)=0 \quad \text { for all } \alpha \in \Delta^{+}(\mathfrak{l}), \quad \text { and } \quad\left(\delta, \alpha_{s}\right) \neq 0 .
\end{aligned}
$$

Consider the category $\mathfrak{g}$-Mod- $T$ of $\mathbb{Z}_{2}$-graded $\mathrm{U}(\mathfrak{g})-T$ bimodules such that the left action of $\mathbb{C} \subset \mathrm{U}(\mathfrak{g})$ and the right action of $\mathbb{C} \subset T$ agree. The $\mathbb{Z}_{2}$-grading of the objects is compatible with the $\mathbb{Z}_{2}$-grading of $U(\mathfrak{g})$. All the morphisms in the category preserve this grading, that is, they are homogeneous of degree 0 . Obviously, $\mathfrak{g}$-Mod- $T$ is an abelian category. For simplicity we shall refer to an object in $\mathfrak{g}$-Mod- $T$ as a $\mathfrak{g}$ - $T$-module.

Let us now fix once for all a morphism $\phi$ satisfying (2.2). Given every object $M$ in the category $\mathfrak{g}$-Mod- $T$, we define the deformed weight space of weight $\mu \in \mathfrak{h}^{*}$ by

$$
M_{\mu}=\{m \in M \mid h m=\mu(h) m+m \phi(h), \forall h \in \mathfrak{h}\} .
$$

Similar to [30], we let $\mathcal{O}^{\mathfrak{p}}(T)$ be the full subcategory of $\mathfrak{g}$-Mod-T such that each object $M$ in $\mathcal{O}^{\mathfrak{p}}(T)$

- is finitely generated over $\mathrm{U}(\mathfrak{g}) \otimes_{\mathbb{C}} T$;

- decomposes into the direct sum of deformed weight spaces $M=\bigoplus_{\mu} M_{\mu}$; and

- is locally $\mathrm{U}(\mathfrak{p})$-finite.

Here local $\mathfrak{p}$-finiteness means that for any $v \in M, \mathrm{U}(\mathfrak{p}) v$ is a $\mathrm{U}(\mathfrak{p})$-submodule of finite complex dimension.

One can easily show that $\mathcal{O}^{\mathfrak{p}}(T)$ is closed under taking submodules and finite direct sums. It is well known that the power series ring is Noetherian, and it is also easy to show (say, by using [7, Proposition I.8.17]) that $U(\mathfrak{g})$ is Noetherian. Thus $\mathcal{O}^{\mathfrak{p}}(T)$ is also closed under taking quotients. It then immediately follows that $\mathcal{O}^{\mathfrak{p}}(T)$, being a full subcategory of the abelian category $\mathfrak{g}$-Mod- $T$, is an abelian category.

The generalised Verma modules are distinguished objects of $\mathcal{O}^{\mathfrak{p}}(T)$, which we now discuss. We need the following easy result.

Lemma 2.1. Corresponding to each $\phi$ with property (2.2), there exists a $\mathfrak{p}$-action on $T$ defined, for all $f \in T$, by

$$
h f=f \phi(h) \quad \text { for all } h \in \mathfrak{h}, \quad X f=0 \quad \text { for all } X \in \mathfrak{g}_{\alpha} \subset \mathfrak{p} .
$$

Proof. This immediately follows from property (2.2) of the map $\phi$. 
For any $\lambda \in P_{0}^{+}$, let $L^{0}(\lambda)$ be the irreducible $\mathfrak{p}$-module with highest weight $\lambda$. Then $L^{0}(\lambda)$ is finite-dimensional. Introduce the $\mathfrak{p}$-module $L_{T}^{0}(\lambda)=L^{0}(\lambda) \otimes_{\mathbb{C}} T$ with $\mathfrak{p}$ acting diagonally. This is also a $\mathfrak{p}-T$-bimodule with $T$ acting on the right by multiplication on the factor $T$. Now we define the generalised Verma module (a $\mathfrak{g}-T$-bimodule) with highest weight $\lambda$ by

$$
K_{T}(\lambda):=\mathrm{U}(\mathfrak{g}) \otimes_{\mathrm{U}(\mathfrak{p})} L_{T}^{0}(\lambda)=\mathrm{U}(\mathfrak{g}) \otimes_{\mathrm{U}(\mathfrak{p})}\left(L^{0}(\lambda) \otimes_{\mathbb{C}} T\right),
$$

where $T$ acts on the last factor by multiplication. Note that $K_{T}(\lambda)$ is a free $T$-module. If $\mathfrak{g}$ is a type I Lie superalgebra, we call $K_{T}(\lambda)$ the deformed Kac module with highest weight $\lambda$. In this case, $K_{T}(\lambda)$ has finite rank over $T$.

\subsection{Generalised Jantzen filtration}

Keep the notation from the last subsection. Denote by $\theta$ the $\mathbb{C}$-linear anti-involution of $\mathfrak{g}$ which maps $\mathfrak{g}_{\alpha}$ to $\mathfrak{g}_{-\alpha}$ for any root space and restricts to the identity map on $\mathfrak{h}$. It extends uniquely to an anti-involution on the universal enveloping algebra $\mathrm{U}(\mathfrak{g})$. Construct a $T$ bilinear form

$$
\langle,\rangle_{0}: L_{T}^{0}(\lambda) \times L_{T}^{0}(\lambda) \rightarrow T
$$

satisfying the following conditions:

$$
\begin{aligned}
& \left\langle x m, m^{\prime}\right\rangle_{0}=\left\langle m, \theta(x) m^{\prime}\right\rangle_{0} \quad \text { for all } m, m^{\prime} \in L_{T}^{0}(\lambda), x \in \mathrm{U}(\mathfrak{l}), \\
& \langle v \otimes 1, v \otimes 1\rangle_{0}=1 \quad \text { for a fixed highest weight vector } v \neq 0 \text { of } L^{0}(\lambda) .
\end{aligned}
$$

Such a form exists, is unique and is nondegenerate in the sense that $\left\langle m, L_{T}^{0}(\lambda)\right\rangle_{0}=\{0\}$ if and only if $m=0$. This follows from the existence of a $\mathbb{C}$-bilinear form on $L^{0}(\lambda)$ with similar properties. We now define a $T$-bilinear form

$$
\langle,\rangle: K_{T}(\lambda) \times K_{T}(\lambda) \rightarrow T
$$

by requiring

- $\left\langle 1 \otimes v^{\prime}, 1 \otimes v^{\prime \prime}\right\rangle=\left\langle v^{\prime}, v^{\prime \prime}\right\rangle_{0}$ for all $v^{\prime}, v^{\prime \prime} \in L_{T}^{0}(\lambda)$

- $\left\langle x m, m^{\prime}\right\rangle=\left\langle m, \theta(x) m^{\prime}\right\rangle$ for all $m, m^{\prime} \in K_{T}(\lambda)$ and $x \in \mathrm{U}(\mathfrak{g})$.

We have the following result.

Lemma 2.2. Assume that the morphism $\phi: \mathrm{U}(\mathfrak{h}) \rightarrow T$ is given by (2.3). Then for any $\lambda \in P_{0}^{+}$, the $\mathrm{U}(\mathfrak{g})$-contravariant $T$-bilinear form $(2.7)$ on $K_{T}(\lambda)$ is nondegenerate.

Proof. Let $z$ be an element of the centre $Z(\mathfrak{g})$ of $\mathrm{U}(\mathfrak{g})$. Then $z$ acts on $K_{T}(\lambda)$ by a scalar $\chi_{\lambda, T}(z) \in T$. In fact $\chi_{\lambda, T}: Z(\mathfrak{g}) \rightarrow T, z \mapsto \chi_{\lambda, T}(z)$, defines a $\mathbb{C}$-algebra homomorphism.

Call a nonzero $\mathrm{U}(\mathfrak{l})$ highest weight vector $v_{\mu} \in K_{T}(\lambda)_{\mu}$ a primitive vector if $v_{\mu}$ does not belong to the $\mathrm{U}(\mathfrak{g})-T$-submodule $V^{\prime}$ generated by $\mathfrak{u} v_{\mu}$. It is important to observe that if the kernel of the form (2.7) is nontrivial, it must contain at least one primitive vector $v_{\mu}$ with $\mu \neq \lambda$. Now $v_{\mu}+V^{\prime}$ is a $\mathrm{U}(\mathfrak{g})$-highest weight vector in the quotient $\mathrm{U}(\mathfrak{g})$ - $T$-module 
$K_{T}(\lambda) / V^{\prime}$. Thus each $z \in Z(\mathfrak{g})$ acts on $K_{T}(\lambda) / V^{\prime}$ by a scalar $\chi_{\mu, T}(z) \in T$, and we have $\chi_{\mu, T}(z)=\chi_{\lambda, T}(z)$ for all $z \in Z(\mathfrak{g})$.

Since $\phi: \mathrm{U}(\mathfrak{h}) \rightarrow T$ is defined by (2.3), $\chi_{\lambda, T}(Z(\mathfrak{g}))$ and $\chi_{\mu, T}(Z(\mathfrak{g}))$ are contained in the subring of $T$ consisting of polynomials. We may specialise $t$ to an arbitrary complex number $c$ to obtain $\mathbb{C}$-algebra homomorphisms

$$
\begin{aligned}
& \chi_{\lambda_{c}}: Z(\mathfrak{g}) \rightarrow \mathbb{C}, \quad z \mapsto \chi_{\lambda_{c}}(z)=\left.\chi_{\lambda, T}(z)\right|_{t=c}, \\
& \chi_{\mu_{c}}: Z(\mathfrak{g}) \rightarrow \mathbb{C}, \quad z \mapsto \chi_{\mu_{c}}(z)=\left.\chi_{\mu, T}(z)\right|_{t=c},
\end{aligned}
$$

where $\lambda_{c}=\lambda+c \delta$ and $\mu_{c}=\mu+c \delta$, and obviously $\chi_{\lambda_{c}}=\chi_{\mu_{c}}$. Now the weights $\lambda_{c}, \mu_{c}$ have the following properties:

- $\lambda_{c}, \mu_{c} \in P_{0}^{+}$;

- $\lambda_{c}-\mu_{c}=\lambda-\mu=\sum_{\alpha \in B} \alpha$ for some nonempty subset $B$ of $\Delta(\mathfrak{u})$;

- there exists $w$ in the Weyl group of $\mathfrak{g}$ such that $\mu_{c}+\rho=w\left(\lambda_{c}+\rho\right)$.

The last condition is required by $\chi_{\lambda_{c}}=\chi_{\mu_{c}}$ and the fact that $\lambda_{c}$ is a typical weight for appropriate values of $c$. Because of the second condition, $w$ cannot be the identity element 1.

For type I Lie superalgebras, one can easily see that $w\left(\lambda_{c}+\rho\right)-\rho$ cannot belong to $P_{0}^{+}$for any $w \neq 1$. For type II Lie superalgebras, there can exist Weyl group elements $w \neq 1$ rendering $w\left(\lambda_{c}+\rho\right)-\rho$ dominant with respect to $\mathfrak{l}$. However, in this case, $\lambda_{c}+\rho-w\left(\lambda_{c}+\rho\right)$ will depend on $c$ linearly, thus cannot be equal to $\sum_{\alpha \in B} \alpha$ for any $B$. Therefore, we conclude that there cannot exist any $\mu_{c}$ satisfying all the conditions. This implies that the kernel of the form (2.7) is trivial.

Remark 2.3. Lemma 2.2 can be proven by a direct computation if $\mathfrak{g}$ is type I. Let $X_{-\alpha} \neq 0$ be a root vector in $\overline{\mathfrak{u}}$ with root $-\alpha$, where $\alpha \in \Delta(\mathfrak{u})$. Given any order on $\Delta(\mathfrak{u})$, we set

$$
D=\prod_{\alpha \in \Delta(\mathfrak{u})} X_{-\alpha}, \quad \text { with factors ordered by the order on } \Delta(\mathfrak{u}) .
$$

Then $\mathfrak{g}_{\beta}$, for all $\beta \in \Delta(\mathfrak{l})$, commutes with $D$. Any $m \neq 0$ in $K_{T}(\lambda)$ can be mapped, by applying $X_{-\alpha}(\alpha \in \Delta(\mathfrak{u}))$, to some nonzero vector $m^{\prime}$ in the l-T-submodule generated by $D(v \otimes 1)$, where $v$ is the highest weight vector of $L^{0}(\lambda)$ chosen in (2.6). Using $\mathfrak{l}$, we can always map $m^{\prime}$ to $D(v \otimes f)$ for some nonzero $f \in T$. Now a computation gives

$$
\begin{aligned}
& \langle D(v \otimes f), D(v \otimes g)\rangle=f g \chi_{0}(\lambda), \quad f, g \in T, \\
& \chi_{0}(\lambda)=\prod_{\alpha \in \Delta(\mathfrak{u})}((\lambda+\rho, \alpha)+t(\delta, \alpha)) .
\end{aligned}
$$

Clearly $\chi_{0}(\lambda)$ is nonzero and so is also $f g \chi_{0}(\lambda)$.

Remark 2.4. Hereafter we shall take $\phi$ to be given by (2.3). 
For each $i \in \mathbb{Z}_{+}$, we define

$$
K_{T}^{i}(\lambda)=\left\{m \in K_{T}(\lambda) \mid\left\langle m, K_{T}(\lambda)\right\rangle \subset t^{i} \mathbb{C}[[t]]\right\} .
$$

Clearly the $K_{T}^{i}(\lambda)$ are $\mathfrak{g}-T$-submodules of $K_{T}(\lambda)$, which give rise to the descending filtration for $K_{T}(\lambda)$

$$
K_{T}(\lambda)=K_{T}^{0}(\lambda) \supset K_{T}^{1}(\lambda) \supset K_{T}^{2}(\lambda) \supset \cdots
$$

Let us consider the specialisation of $\mathcal{O}^{\mathfrak{p}}(T)$ to the parabolic category $\mathcal{O}^{\mathfrak{p}}$ of $\mathfrak{g}$ over the complex number field. Regard $\mathbb{C}$ as a $T$-module with $f(t) \in \mathbb{C}[[t]]$ acting by multiplication by $f(0)$. Let $\mathcal{R}: \mathcal{O}^{\mathfrak{p}}(T) \rightarrow \mathcal{O}^{\mathfrak{p}}$ be the specialisation functor which sends an object $M$ in $\mathcal{O}^{\mathfrak{p}}(T)$ to $M \otimes_{T} \mathbb{C}$ in $\mathcal{O}^{\mathfrak{p}}$, and a morphism $\psi: M \rightarrow N$ to

$$
\mathcal{R}(\psi): M \otimes_{T} \mathbb{C} \rightarrow N \otimes_{T} \mathbb{C}, \quad \mathcal{R}(\psi)\left(m \otimes_{T} c\right)=\psi(m) \otimes_{T} c .
$$

Now consider the filtration (2.10) of $K_{T}(\lambda)$ under the functor $\mathcal{R}$. Denote $K(\lambda)=$ $K_{T}(\lambda) \otimes_{T} \mathbb{C}$ and $K^{i}(\lambda)=K_{T}^{i}(\lambda) \otimes_{T} \mathbb{C}$. Applying the specialisation functor $\mathcal{R}$ to $(2.10)$ we obtain the descending filtration

$$
K(\lambda)=K^{0}(\lambda) \supset K^{1}(\lambda) \supset K^{2}(\lambda) \supset \cdots,
$$

which is a generalisation of the Jantzen filtration for Verma modules of Lie algebras to the case of generalised Verma modules of Lie superalgebras. For simplicity, we shall refer to it as the Jantzen filtration for $K(\lambda)$. We also define the consecutive quotients of the Jantzen filtration:

$$
K_{i}(\lambda)=K^{i}(\lambda) / K^{i+1}(\lambda), \quad i=0,1,2, \ldots
$$

We have the following result.

Lemma 2.5. For any $\lambda \in P_{0}^{+}$,

(1) the submodule $K^{1}(\lambda)$ is the unique maximal proper submodule of $K(\lambda)$;

(2) each $K_{i}(\lambda)$ admits a nondegenerate contravariant bilinear form.

Proof. Part (1) is clear. For part (2), we extract a contravariant bilinear form $(,)_{i}$ on $K_{i}(\lambda)$ from the form $\langle$,$\rangle on K_{T}(\lambda)$ (defined by (2.7)) in the following way. For any $w, w^{\prime} \in K_{i}(\lambda)$, we let $w_{T}$ and $w_{T}^{\prime}$ be elements in $K_{T}^{i}(\lambda)$ such that $w_{T} \otimes_{T} 1, w_{T}^{\prime} \otimes_{T} 1 \in$ $K^{i}(\lambda)$ are representatives of $w$ and $w^{\prime}$ respectively. Then set

$$
\left(w, w^{\prime}\right)_{i}=\lim _{t \rightarrow 0} t^{-i}\left\langle w_{T}, w_{T}^{\prime}\right\rangle
$$

which defines a contravariant bilinear form on $K_{i}(\lambda)$ since $\langle$,$\rangle is contravariant. It follows$ from general facts on nondegenerate bilinear forms $[22, \S 5.1]$ (see also $[20, \S 5.6]$ ) that $(,)_{i}$ is nondegenerate. 


\subsection{Main problems to be addressed}

Now we describe in more precise terms the main problems to be addressed in this paper and its sequel.

Recall that a descending filtration of a module $M$

$$
M=M^{0} \supset M^{1} \supset M^{2} \supset \cdots \supset M^{l} \supset M^{l+1}=\{0\}
$$

is called a Loewy filtration if the consecutive quotients $M_{i}=M^{i+1} / M^{i}$ are all semisimple, and its length $l$ is minimal. The socle filtration and radical filtration are distinguished examples of Loewy filtrations. A module is called rigid if it has a unique Loewy filtration. This happens if and only if the socle filtration and the radical filtration coincide.

One of the main problems to be addressed is whether the following statement is true for Lie superalgebras.

Statement 2.6. For any $\lambda \in P_{0}^{+}$, the Jantzen filtration is the unique Loewy filtration of the generalised Verma module $K(\lambda)$.

If the statement holds, then it implies in particular that $K(\lambda)$ is rigid. Note that Kac modules of $\mathfrak{g l}_{m \mid n}$ are known to be rigid [9, IV].

Let $L(\lambda)$ be an irreducible $\mathfrak{g}$-module with highest weight $\lambda \in P_{0}^{+}$, which restricts to a module over the nilradical of the parabolic subalgebra $\mathfrak{p}$. Let $H^{i}(\mathfrak{u}, L(\lambda))$ be the $i$-th Lie superalgebra cohomology group of $\mathfrak{u}$ with coefficients in $L(\lambda)$, which admits a semisimple l-action. For $\mu \in P_{0}^{+}$, we let $L^{0}(\mu)$ be the irreducible l-module with highest weight $\mu$. The following generalised Kazhdan-Lusztig polynomials in the indeterminate $q$ were introduced in [28]:

$$
p_{\lambda \mu}(q)=\sum_{i=0}^{\infty}(-q)^{i}\left[H^{i}(\mathfrak{u}, L(\lambda)): L^{0}(\mu)\right],
$$

where $\left[H^{i}(\mathfrak{u}, L(\lambda)): L^{0}(\mu)\right]$ is the multiplicity of $L^{0}(\mu)$ in $H^{i}(\mathfrak{u}, L(\lambda))$. It is a standard fact that $\left[H^{i}(\mathfrak{u}, L(\lambda)): L^{0}(\mu)\right]=\operatorname{dim} \operatorname{Ext}^{i}(K(\mu), L(\lambda))$, where $\operatorname{Ext}^{i}$ are defined in the category $\mathcal{O}^{\mathfrak{p}}$.

Choose a linear order on $P_{0}^{+}$compatible with the usual partial order defined by the positive roots. Then the matrix $P(q)=\left(p_{\lambda \mu}(q)\right)_{\lambda, \mu \in P_{0}^{+}}$is upper triangular with diagonal entries being 1. Let $A(q)=\left(a_{\lambda \mu}(q)\right)_{\lambda, \mu \in P_{0}^{+}}$be the inverse matrix of $P(q)$, and refer to $a_{\lambda \mu}(q)$ as the inverse Kazhdan-Lusztig polynomials of $\mathfrak{g}$. For any $\lambda, \mu \in P_{0}^{+}$, we also define

$$
J_{\lambda \mu}(q)=\sum_{i=0}^{\infty} q^{i}\left[K_{i}(\lambda): L(\mu)\right], \quad i=0,1, \ldots,
$$

where $\left[K_{i}(\lambda), L(\mu)\right]$ denotes the multiplicity of the irreducible $\mathfrak{g}$-module $L(\mu)$ in $K_{i}(\lambda)$. For easy reference, we call $J_{\lambda \mu}(q)$ Jantzen polynomials.

The other main problem to be addressed is whether the following statement holds for Lie superalgebras. 
Statement 2.7. For any $\lambda, \mu \in P_{0}^{+}$, the Jantzen polynomials $J_{\lambda \mu}(q)$ coincide with the inverse Kazhdan-Lusztig polynomials $a_{\lambda \mu}(q)$.

In the present paper we shall prove that both statements are true for type I Lie superalgebras.

\section{Jantzen filtration of type I Lie superalgebras}

In this section we study the Jantzen filtration for Kac modules of type I Lie superalgebras. Keep the notation of the last section, and let $\mathfrak{g}$ denote a type I Lie superalgebra in the remainder of the paper.

Let us first establish the following result.

Lemma 3.1. Assume that $\mathfrak{g}$ is a Lie superalgebra of type $I$, and the weight $\lambda \in P_{0}^{+}$has degree of atypicality $\sharp(\lambda)=r$. Let $K_{T}(\lambda)_{\lambda-2 \rho_{1}}$ be the deformed weight space of weight $\lambda-2 \rho_{1}$ in the deformed Kac module $K_{T}(\lambda)$. Then

$$
K_{T}(\lambda)_{\lambda-2 \rho_{1}} \subset K_{T}^{r}(\lambda), \quad K_{T}(\lambda)_{\lambda-2 \rho_{1}} \not \subset K_{T}^{r+1}(\lambda) .
$$

Proof. In the case of a type I Lie superalgebra, the deformed weight space $K_{T}(\lambda)_{\lambda-2 \rho_{1}}$ of weight $\lambda-2 \rho_{1}$ in $K_{T}(\lambda)$ is $D(v \otimes T)$ (notation as in Remark 2.3). All $K_{T}(\lambda)_{\mu}$ with $\mu \neq \lambda-2 \rho_{1}$ are orthogonal to $D(v \otimes T)$ with respect to the form (2.7). Now for any $m, n \in D(v \otimes T)$, we have $\langle m, n\rangle \in \chi_{0}(\lambda) \mathbb{C}[[t]]$ by (2.9). If the degree of atypicality of $\lambda \in P_{0}^{+}$is $r$, then $\chi_{0}(\lambda) \in t^{r} \mathbb{C}[[t]]$ but $\chi_{0}(\lambda) \notin t^{r+1} \mathbb{C}[[t]]$. This proves the lemma.

Using the lemma, we can easily prove the following result on the length of the Jantzen filtration.

Theorem 3.2. Assume that $\mathfrak{g}$ is of type $I$, and let $\lambda \in P_{0}^{+}$. Then the Jantzen filtration for the Kac module $K(\lambda)$ has length $r=\sharp(\lambda)$, that is,

$$
K(\lambda)=K^{0}(\lambda) \supset K^{1}(\lambda) \supset K^{2}(\lambda) \supset \cdots \supset K^{r}(\lambda) \supset\{0\}
$$

with $K^{r}(\lambda) \neq\{0\}$.

Proof. Let $\bar{L}(\lambda)$ be the submodule of $K(\lambda)=\mathcal{R}\left(K_{T}(\lambda)\right)$ generated by $D(v \otimes 1) \otimes 1$, where $v$ is the highest weight vector of $L^{0}(\lambda)$ chosen in (2.6). We will call $\bar{L}(\lambda)$ the bottom composition factor of $K(\lambda)$. It immediately follows from Lemma 3.1 that $K^{r}(\lambda) \supset \bar{L}(\lambda)$ and $K^{r+1}(\lambda) \not \supset \bar{L}(\lambda)$. Since every nonzero submodule of $K(\lambda)$ must contain $\bar{L}(\lambda)$, we necessarily have $K^{r+1}(\lambda)=\{0\}$.

\subsection{The case of $\mathfrak{o s p}_{2 \mid 2 n}$}

Using Theorem 3.2, we can prove the following result for the Lie superalgebra $\mathfrak{o s p}_{2 \mid 2 n}$.

Theorem 3.3. Both Statements 2.6 and 2.7 are true for Jantzen filtrations for the Kac modules of $\mathfrak{o s p}_{2 \mid 2 n}$. 
Proof. Consider the Kac module $K(\lambda)$ for $\mathfrak{o s p}_{2 \mid 2 n}$ with highest weight $\lambda \in P_{0}^{+}$. If $\lambda$ is typical, $K(\lambda)$ is irreducible, and the theorem is obviously true.

If $\lambda$ is atypical, we necessarily have $\sharp(\lambda)=1$, and $K(\lambda)$ has the composition series $K(\lambda) \supset \bar{L}(\lambda) \supset\{0\}$ of length 2 , which coincides with the Jantzen filtration by Theorem 3.2. Since $K(\lambda)$ is indecomposable, the composition series is the unique Loewy filtration in this case. The rigidity of $K(\lambda)$ immediately follows.

The highest weight of the irreducible submodule $\bar{L}(\lambda)$ can be determined in the following way. Denote by $\gamma$ the unique atypical (positive) root of $\lambda$. Let $k$ be the smallest positive integer such that $\mu=\lambda-k \gamma$ is l-regular in the sense that $(\mu+\rho, \alpha) \neq 0$ for all $\alpha \in \Delta^{+}(\mathfrak{l})$. Then there exists a unique element $w$ in the Weyl group of $\mathfrak{g}$ such that $\lambda^{(1)}=w(\mu+\rho)-\rho \in P_{0}^{+}$. The highest weight of $\bar{L}(\lambda)$ is $\lambda^{(1)}$.

Define $\lambda^{(i+1)}$ for $i \geq 0$ recursively by $\lambda^{(i+1)}=\left(\lambda^{(i)}\right)^{(1)}$. By [40, Corollary 6.4.], $p_{\lambda \lambda^{(i)}}(q)=(-q)^{i}$ and $p_{\lambda \mu}=0$ if $\mu \neq \lambda^{(i)}$ for any $i$. We can easily work out the corresponding inverse Kazhdan-Lusztig polynomials:

$$
a_{\lambda \lambda}=1, \quad a_{\lambda \lambda(1)}=q, \quad \text { rest }=0 .
$$

They clearly agree with the polynomials $J_{\lambda \mu}(q)$, proving the theorem.

\subsection{Statement 2.6 for $\mathfrak{g l}_{m \mid n}$}

Let us first introduce some necessary notation. Denote by $e_{a b}(a, b=1, \ldots, m+n)$ the matrix units of size $(m+n) \times(m+n)$, which form a basis of $\mathfrak{g}=\mathfrak{g l}_{m \mid n}$. Let $\mathfrak{h}$ be the subalgebra of the diagonal matrices. Choose a basis $\epsilon_{-m}, \ldots, \epsilon_{-1}, \epsilon_{1}, \ldots, \epsilon_{n}$ for $\mathfrak{h}^{*}$ such that

$$
\epsilon_{a}\left(e_{i i}\right)=\delta_{a, i-m-1} \quad \text { if } 1 \leq i \leq m, \quad \epsilon_{a}\left(e_{j j}\right)=\delta_{a, j-m} \quad \text { if } m<j \leq m+n .
$$

The bilinear form on $\mathfrak{g}$ defined by the supertrace induces a bilinear form $\left(\right.$, , on $\mathfrak{h}^{*}$ such that $\left(\epsilon_{a}, \epsilon_{b}\right)=\operatorname{sign}(a) \delta_{a b}$, where $\operatorname{sign}(a)=a /|a|$. Set $\delta_{i}=\epsilon_{-i}$ for $1 \leq i \leq m$, and write any $\lambda \in \mathfrak{h}^{*}$ in terms of its coordinates as

$$
\lambda=\sum_{i=1}^{m} \lambda_{m+1-i} \delta_{i}+\sum_{j=1}^{n} \lambda_{m+j} \epsilon_{j}=\left(\lambda_{1} \ldots \lambda_{m} \mid \lambda_{m+1} \ldots \lambda_{m+n}\right) .
$$

We similarly write $\lambda+\rho=\left(\lambda_{1}^{\rho} \ldots \lambda_{m}^{\rho} \mid \lambda_{m+1}^{\rho} \ldots \lambda_{m+n}^{\rho}\right)$.

Remark 3.4. The unusual labelling of the basis elements $\delta_{i}$ and $\epsilon_{j}$ of $\mathfrak{h}^{*}$ will become convenient when we discuss weight diagrams in Section 5.1.

Let us choose the standard Borel subalgebra $\mathfrak{b} \subset \mathfrak{g}$ consisting of upper triangular matrices, which contains the standard Cartan subalgebra $\mathfrak{h}$. Then the simple roots of $\mathfrak{g}$ are given by $\delta_{m}-\delta_{m-1}, \ldots, \delta_{2}-\delta_{1}, \delta_{1}-\epsilon_{1}, \epsilon_{1}-\epsilon_{2}, \ldots, \epsilon_{n-1}-\epsilon_{n}$; the set of positive even roots and the set of positive odd roots are respectively given by

$$
\begin{aligned}
& \Delta_{0}^{+}=\left\{\delta_{a}-\delta_{b}, \epsilon_{a^{\prime}}-\epsilon_{b^{\prime}} \mid m \geq a>b \geq 1,1 \leq a^{\prime}<b^{\prime} \leq n\right\}, \\
& \Delta_{1}^{+}=\left\{\delta_{a}-\epsilon_{b} \mid 1 \leq a \leq m, 1 \leq b \leq n\right\} .
\end{aligned}
$$


Denote by $\mathfrak{g}_{+1}$ (resp. $\mathfrak{g}_{-1}$ ) the nilpotent subalgebra spanned by the odd positive (resp. negative) root spaces. Then $\mathfrak{g}=\mathfrak{g}_{-1} \oplus \mathfrak{g}_{0} \oplus \mathfrak{g}_{+1}$ with $\mathfrak{g}_{0} \cong \mathfrak{g l}_{m} \oplus \mathfrak{g l}_{n}$. We also denote by $\mathfrak{g}^{+}$(resp. $\mathfrak{g}^{-}$) the subalgebra of strictly upper (resp. lower) triangular matrices.

We define a total order on $\Delta_{1}^{+}$by

$$
\delta_{a}-\epsilon_{b}<\delta_{a^{\prime}}-\epsilon_{b^{\prime}} \Leftrightarrow a+b>a^{\prime}+b^{\prime}, \text { or } a+b=a^{\prime}+b^{\prime}, a>a^{\prime} .
$$

We also introduce the sets

$$
X=\sum_{i=1}^{m} \mathbb{Z} \delta_{i}+\sum_{j=1}^{n} \mathbb{Z}_{+} \epsilon_{j}, \quad X^{+}=P_{0}^{+} \cap X .
$$

Now the special linear algebra $\mathfrak{s l}_{m \mid n}$ is the subalgebra of $\mathfrak{g}=\mathfrak{g l}_{m \mid n}$ consisting of matrices with vanishing supertrace. All information on the category $\mathcal{O}^{\mathfrak{p}}$ of $\mathfrak{s l}_{m \mid n}$ can be extracted from the corresponding category of $\mathfrak{g l}_{m \mid n}$ by tensoring with one-dimensional modules. In particular, if $\zeta \in \mathfrak{h}^{*}$ satisfies the condition $(\zeta, \beta)=0$ for all $\beta \in \Delta^{+}$, by using the tensor identity we easily see that $L(\zeta) \otimes_{\mathbb{C}} K_{T}(\lambda)=K_{T}(\lambda+\zeta)$ as $\mathfrak{g}-T$-modules. The following result immediately follows.

Lemma 3.5. The isomorphism $L(\zeta) \otimes K(\lambda) \stackrel{\sim}{\rightarrow} K(\lambda+\zeta)$ of $\mathfrak{g l}_{m \mid n}$-modules maps the tensor product

$$
L(\zeta) \otimes K(\lambda)=L(\zeta) \otimes K^{0}(\lambda) \supset L(\zeta) \otimes K^{1}(\lambda) \supset \cdots \supset L(\zeta) \otimes K^{\#(\lambda)}(\lambda) \supset\{0\}
$$

of $L(\zeta)(\operatorname{dim} L(\zeta)=1)$ with the Jantzen filtration of $K(\lambda)$ to the Jantzen filtration for $K(\zeta+\lambda)$ :

$$
K(\lambda)=K^{0}(\lambda) \supset K^{1}(\lambda) \supset \cdots \supset K^{\#(\lambda)}(\lambda) \supset\{0\} .
$$

Furthermore, a $\zeta$ can always be chosen to make the identity matrix in $\mathfrak{g}$ act on $K(\lambda+\zeta)$ by zero. Thus one may regard $K(\lambda+\zeta)$ as an $\mathfrak{s l}_{m \mid n}$-module, and (3.3) its Jantzen filtration.

Therefore, we shall work only with the general linear superalgebra $\mathfrak{g}=\mathfrak{g l}_{m \mid n}$ in the remainder of the paper.

Recall that the inverse Kazhdan-Lusztig polynomials for type I Lie superalgebras have all been determined explicitly (see [8] and [39, Conjecture 4]). Each $a_{\lambda \mu}(q)$ is either zero, or a positive power of $q$. This in particular implies that the multiplicity of a simple module $L(\mu)$ in a Kac module $K(\lambda)$ is at most 1 . This fact will be used in a crucial way in the proof of the following result.

Theorem 3.6. Statement 2.6 holds for $\mathfrak{g l}_{m \mid n}$, namely, the Jantzen filtration for any Kac module $K(\lambda)$ with $\lambda \in P_{0}^{+}$is the unique Loewy filtration.

Proof. We first show that the consecutive quotients of the Jantzen filtration are semisimple. Let $L$ be an irreducible submodule in $K_{i}(\lambda)$, and denote $L^{\perp}=\left\{w \in K_{i}(\lambda) \mid\right.$ $\left.(w, L)_{i}=\{0\}\right\}$, where $(,)_{i}$ is the nondegenerate contravariant bilinear form on $K_{i}(\lambda)$ discussed in Lemma 2.5. We want to show that $L^{\perp} \cap L=\{0\}$, which implies $K_{i}(\lambda)=$ $L^{\perp} \oplus L$. Then by repeating the argument for $L^{\perp}$ we can prove the semisimplicity of consecutive quotients of the Jantzen filtration. 
Now since $(,)_{i}$ induces a contravariant bilinear form $L \times\left(K_{i}(\lambda) / L^{\perp}\right) \rightarrow \mathbb{C}$, we must have $K_{i}(\lambda) / L^{\perp} \cong L$. Since the multiplicity of $L$ in $K_{i}(\lambda)$ must be 1 [8, 39], $L^{\perp}$ cannot have any composition factor isomorphic to $L$. This in particular rules out the possibility that $L^{\perp} \supset L$. Hence $L^{\perp} \cap L=\{0\}$ since $L$ is irreducible.

We now show that the Jantzen filtration is a Loewy filtration. Recall that for a module $V$ for $\mathfrak{g}=\mathfrak{g l}_{m \mid n}$, a nonzero $\mathfrak{g}_{0}$-highest weight vector $v \in V$ is called a primitive vector if there exists a $\mathfrak{g}$-submodule $W$ of $V$ such that $v \notin W$ but $\mathfrak{g}_{+1} v \in W$. If we can take $W=0$, then $v$ is called a strongly primitive vector or $\mathfrak{g}$-highest weight vector. The weight of a primitive vector is called a primitive weight, and that of a strongly primitive vector a strongly primitive weight or a $\mathfrak{g}$-highest weight. Let $v$ and $v^{\prime}$ be nonzero primitive vectors with distinct weights. We say that $v^{\prime}$ is generated by $v$ if $v^{\prime} \in \mathrm{U}(\mathfrak{g}) v$. We have the following result.

Lemma 3.7 (see also Lemma 4.6). In every Kac module $K(\lambda)$ with $\sharp(\lambda)=r$, there exist $r+1$ nonzero primitive vectors $v_{\lambda}=v_{0}, v_{1}, \ldots, v_{r}$ with distinct primitive weights $\mu_{i}=\operatorname{wt}\left(v_{i}\right)$, where $\mu_{0}=\lambda$, such that $v_{k}$ can be generated by $v_{k-1}$ for each $k=1, \ldots, r$.

The proof of the lemma will be given in Section 4.2.

It immediately follows from Lemma 3.7 that $r$ is the shortest possible length of all the filtrations of $K(\lambda)$ with semisimple consecutive quotients. Thus the Jantzen filtration is a Loewy filtration.

Finally, we show that the Jantzen filtration of the Kac module is the unique Loewy filtration. Let $\mathcal{O}_{\text {int }}^{\mathfrak{p}}$ be the full subcategory of the parabolic category $\mathcal{O}^{\mathfrak{p}}$ of $\mathfrak{g l}_{m \mid n}$ such that each object has only weights in $X$. Then $\mathcal{O}_{\text {int }}^{\mathfrak{p}}$ is equivalent to a category of modules of a generalised Khovanov algebra [9]. It is one of the consequences of this equivalence of categories that Kac modules of $\mathfrak{g l}_{m \mid n}$ in $\mathcal{O}_{\text {int }}^{\mathfrak{p}}$ are rigid [9, IV]. Every Kac module in $\mathcal{O}^{\mathfrak{p}}$ can be turned into an object in $\mathcal{O}_{\text {int }}^{\mathfrak{p}}$ by tensoring with a 1-dimensional module of appropriate weight. It follows from the first part of Lemma 3.5 that every Kac module $K(\lambda)$ for $\lambda \in P_{0}^{+}$is rigid, thus its Jantzen filtration is the unique Loewy filtration. This completes the proof of the theorem.

Remark 3.8. Parabolic BGG categories with multiplicity free generalised Verma modules for semisimple Lie algebras were studied extensively in $[15,6,21]$. The case of $\mathfrak{g l}_{m}$ with a maximal parabolic has been treated in detail in [31] from a modern perspective.

Remark 3.9. In [31, Section 5], Stroppel described a precise connection between diagram algebras introduced in [9] and the category of perverse sheaves on Grassmannians. A realization of diagram algebras as cohomology algebras using the geometry of Springer fibres was found by Stroppel and Webster in [32].

\subsection{Some equivalences of categories}

In this subsection we make some preparations for proving Statement 2.7 for $\mathfrak{g l}_{m \mid n}$. 
3.3.1. Super duality of type A. The material presented here is largely from [13]. Consider the embedding of Lie superalgebras $\mathfrak{g l}_{m \mid N} \hookrightarrow \mathfrak{g l}_{m \mid N+1}$ for each $N$, where the image of $\mathfrak{g l}_{m \mid N}$ consists of matrices with vanishing $(m+N+1)$-th row and $(m+N+1)$-th column. This defines a direct system

$$
\mathfrak{g l}_{m \mid 1} \hookrightarrow \mathfrak{g l}_{m \mid 2} \hookrightarrow \cdots \hookrightarrow \mathfrak{g l}_{m \mid N} \hookrightarrow \mathfrak{g l}_{m \mid N+1} \hookrightarrow \cdots
$$

of Lie superalgebras, and we denote the direct limit by $\mathfrak{g l}_{m \mid \infty}$. Let $\mathfrak{p}_{m \mid N} \supset \mathfrak{b}_{m \mid N} \supset \mathfrak{h}_{m \mid N}$ be the standard parabolic, Borel, and Cartan suablgebras of $\mathfrak{g l}_{m \mid N}$. Then we have the corresponding direct systems of these subalgebras induced by the embedding of $\mathfrak{g l}_{m \mid N}$ in $\mathfrak{g l}_{m \mid N+1}$ for each $N$. Let the direct limits be $\mathfrak{p}_{m \mid \infty}, \mathfrak{b}_{m \mid \infty}$ and $\mathfrak{h}_{m \mid \infty}$ respectively.

To emphasize the dependence on $m$ and $N$, we denote by $X(m \mid N)$ and $X^{+}(m \mid N)$ respectively the subsets of $\mathfrak{h}_{m \mid N}^{*}$ defined by (3.2) (with $N=n$ ). There is the natural $\mathbb{Z}_{+}$-module embedding of $X(m \mid N)$ in $X(m \mid N+1)$ for each $N$, where the image of $X(m \mid N)$ consists of elements with the $(m+N+1)$-th coordinate being zero. Thus we have the direct limits $X(m \mid \infty)$ and $X^{+}(m \mid \infty)$. In particular, when we write an element $\lambda \in X^{+}(m \mid \infty)$ in terms of its coordinate $\lambda=\left(\lambda^{-} \mid \lambda^{+}\right)$in the notation of (3.1) (for $n$ infinite), then $\lambda^{-}$is an $m$-tuple and $\lambda^{+}$, an infinite tuple, is a partition of finite length. For every finite $N$, we shall regard every $X(m \mid N)$ (resp. $\left.X^{+}(m \mid N)\right)$ as the subset of $X(m \mid \infty)$ (resp. $\left.X^{+}(m \mid \infty)\right)$ consisting of the elements $\mu$ satisfying $\mu_{m+N+k}=0$ for all $k>0$.

For each $N$, let $\mathcal{O}_{\text {int }}^{\mathfrak{p}_{m \mid N}}$ be the parabolic category of $\mathfrak{g l}_{m \mid N}$-modules with weights in $X(m \mid N)$. To indicate the $N$ dependence, we denote by $K^{m \mid N}(\lambda)$ and $L^{m \mid N}(\lambda)$ the Kac module and irreducible module with highest weight $\lambda$ respectively. Now $K^{m \mid N}(\lambda)$ (resp. $\left.L^{m \mid N}(\lambda)\right)$ can be embedded in $K^{m \mid N+1}(\lambda)$ (resp. $\left.L^{m \mid N+1}(\lambda)\right)$ as the subspace spanned by the weight vectors with weights $\mu$ satisfying $\mu_{m+N+k}=0$ for all $k>0$. This defines a direct system of modules compatible with the direct system (3.4) of Lie superalgebras. Then $K^{m \mid \infty}(\lambda)$ (resp. $L^{m \mid \infty}(\lambda)$ ) is the direct limit. For each finite $N$, we have an exact functor $\operatorname{tr}_{N}: \mathcal{O}_{\text {int }}^{\mathfrak{p}_{m}} \rightarrow \mathcal{O}_{\text {int }}^{\mathfrak{p}_{m \mid N}}$, the truncation functor, which maps each object to the span of the weight vectors with weights $\mu$ satisfying $\mu_{m+N+k}=0$ for all $k>0$. In particular,

$\operatorname{tr}_{N} K^{m \mid \infty}(\lambda)=K^{m \mid N}(\lambda), \quad \operatorname{tr}_{N} L^{m \mid \infty}(\lambda)=L^{m \mid N}(\lambda), \quad$ if $\lambda \in X^{+}(m \mid N) \subset X^{+}(m \mid \infty)$.

One can define generalised Kazhdan-Lusztig polynomials $p_{\lambda \mu}^{(m \mid N)}(q)$ as in (2.13) and their inverse polynomials $a_{\lambda \mu}^{(m \mid N)}(q)$ for each $N$. Fix $\lambda=\left(\lambda^{-} \mid \lambda^{+}\right)$and $\mu=\left(\mu^{-} \mid \mu^{+}\right)$in $X^{+}(m \mid \infty)$, we may regard them as elements of $X^{+}\left(m \mid N^{\prime}\right)$ for any $N^{\prime}$ greater than the numbers of positive entries in $\lambda^{+}$and $\mu^{+}$. Then

$$
p_{\lambda \mu}^{(m \mid N)}(q)=p_{\lambda \mu}^{\left(m \mid N^{\prime}\right)}(q), \quad a_{\lambda \mu}^{(m \mid N)}(q)=a_{\lambda \mu}^{\left(m \mid N^{\prime}\right)}(q), \quad \text { for all } N>N^{\prime} .
$$

The above discussion can be repeated verbatim for the series of ordinary Lie algebras $\mathfrak{g l}_{m+N}$. Let $\mathfrak{b}_{m+N}$ be the standard Borel subalgebra, and $\mathfrak{h}_{m+N}$ the standard Cartan subalgebra. Let $X(m+N)$ be the subset of $\mathfrak{h}_{m+N}^{*}$ consisting of the elements $\lambda$ satisfying $\lambda\left(e_{i i}\right) \in \mathbb{Z}(i \leq m)$ and $\lambda\left(e_{m+j, m+j}\right) \in \mathbb{Z}_{+}(j \geq m)$, and $X^{+}(m+N)$ be the subset of $X(m+N)$ consisting of the elements which are dominant with respect to the subalgebra $\mathfrak{l}_{m+N}=\mathfrak{g l}_{m} \oplus \mathfrak{g l}_{N}$. Let $\mathfrak{p}_{m+N} \supset \mathfrak{b}_{m+N}$ be the parabolic subalgebra with Levi 
subalgebra $\mathfrak{l}_{m+N}$. Then we have the parabolic category $\mathcal{O}^{\mathfrak{p}_{m+N}}$ of $\mathfrak{g l}_{m+N}$, where every object is a locally $\mathfrak{p}_{m+N}$ finite weight module with weights belonging to $X(m+N)$. Denote by $M^{m+N}(\mu)$ and $L^{m+N}(\mu)$ respectively the generalised Verma module and irreducible module with highest weight $\mu$. In the limit $N \rightarrow \infty$, we have $\mathfrak{g l}_{m+\infty}, \mathfrak{p}_{m+\infty}$, $X(m+\infty), X^{+}(m+\infty)$, etc. We shall also write $\mu \in X_{m+\infty}$ as $\mu=\left(\mu^{-} \mid \mu^{+}\right)$, where $\mu^{-}=\left(\mu_{1} \ldots \mu_{m}\right)$ and $\mu^{+}=\left(\mu_{m+1} \mu_{m+2} \ldots\right)$ with $\mu_{j}=\mu\left(e_{j j}\right)$.

For each finite $N$, we also have the truncation functor $\operatorname{tr}_{N}: \mathcal{O}_{\text {int }}^{\mathfrak{p}_{m+\infty}} \rightarrow \mathcal{O}_{\text {int }}^{\mathfrak{p}_{m+N}}$, which is also an exact functor mapping each object to the span of the weight vectors with weights $\mu$ satisfying $\mu_{m+N+k}=0$ for all $k>0$. In particular,

$$
\operatorname{tr}_{N} M^{m+\infty}(\lambda)=M^{m+N}(\lambda), \quad \operatorname{tr}_{N} L^{m+\infty}(\lambda)=L^{m+N}(\lambda)
$$

for any $\lambda \in X^{+}(m+N) \subset X^{+}(m+\infty)$.

We can define generalised Kazhdan-Lusztig polynomials $k_{\lambda \mu}^{(m+N)}(q)$ as in (2.13) for $\mathfrak{g l}_{m+N}$ by using the cohomology of the nilpotent radical of $\mathfrak{p}_{m+N}$ for each $N$. Then we can also define their inverse polynomials $b_{\lambda \mu}^{(m+N)}(q)$ for each $N$. Fix $\lambda=\left(\lambda^{-} \mid \lambda^{+}\right)$and $\mu=\left(\mu^{-} \mid \mu^{+}\right)$in $X^{+}(m+\infty)$, and regard them as elements of $X^{+}\left(m+N^{\prime}\right)$ for any $N^{\prime}$ greater than the numbers of positive entries in $\lambda^{+}$and $\mu^{+}$. Then

$$
k_{\lambda \mu}^{(m+N)}(q)=k_{\lambda \mu}^{\left(m+N^{\prime}\right)}(q), \quad b_{\lambda \mu}^{(m+N)}(q)=b_{\lambda \mu}^{\left(m+N^{\prime}\right)}(q), \quad \text { for all } N>N^{\prime} .
$$

Given a partition $v$, we denote by $v^{\prime}$ its transpose partition. Then there is the bijection

$$
{ }^{\natural}: X^{+}(m+\infty) \rightarrow X^{+}(m \mid \infty), \quad \lambda=\left(\lambda^{-} \mid \lambda^{+}\right) \mapsto \lambda^{\natural}=\left(\lambda^{-} \mid\left(\lambda^{+}\right)^{\prime}\right) .
$$

We shall also denote the inverse map by ${ }^{\natural}$. It was shown in [13] that for fixed $\lambda$ and $\mu$ in $X^{+}(m+\infty)$,

$$
k_{\lambda \mu}^{(m+N)}(q)=p_{\lambda^{\natural} \mu^{\natural}}^{(m \mid N)}(q), \quad b_{\lambda \mu}^{(m+N)}(q)=a_{\lambda^{\natural} \mu^{\natural}}^{(m \mid N)}(q), \quad N \text { sufficiently large. }
$$

This and other facts in [13] indicated that the following result is true.

Theorem 3.10. There is an equivalence $\mathcal{O}_{\mathrm{int}}^{\mathfrak{p}_{m \mid \infty}} \stackrel{\sim}{\rightarrow} \mathcal{O}_{\mathrm{int}}^{\mathfrak{p}_{m+\infty}}$ of categories, which sends

$$
M^{m+\infty}(\lambda) \rightarrow K^{m \mid \infty}\left(\lambda^{\natural}\right), \quad L^{m+\infty}(\lambda) \rightarrow L^{m \mid \infty}\left(\lambda^{\natural}\right), \quad \text { for } \lambda \in X^{+}(m+\infty) .
$$

The equivalence has since been proven in [11] and [9, IV] using different methods.

Remark 3.11. The "super duality" was first observed in [14] for tensorial representations of the general linear superalgebra. A similar duality for the orthosymplectic Lie superalgebras was recently established in [12]. 
3.3.2. Jantzen filtration under Serganova's equivalence of categories. We shall also require a result of [29], which we now explain. For any $m$ and $n$, we denote by $P_{0}^{+}(m \mid n)$ the set of integral dominant $\mathfrak{g l}_{m \mid n}$-weights defined by (2.1). Given a $\lambda^{(m \mid n)} \in P_{0}^{+}(m \mid n)$, we denote by $\mathcal{O}^{\mathfrak{p}_{m \mid n}}\left(\lambda^{(m \mid n)}\right)$ the full subcategory of the category $\mathcal{O}^{\mathfrak{p}_{m \mid n}}$ for $\mathfrak{g l}_{m \mid n}$-modules with infinitesimal character specified by $\lambda^{(m \mid n)}$. We shall also write $\rho^{(m \mid n)}$ for the $\rho$ of $\mathfrak{g l}_{m \mid n}$ to emphasize the dependence on $m$ and $n$.

If a weight $\lambda^{(m \mid n)}=\left(\lambda_{1}, \ldots, \lambda_{m} \mid \lambda_{m+1}, \ldots, \lambda_{m+n}\right) \in P_{0}^{+}(m \mid n)$ is $r$-fold atypical, there exist $m \geq i_{r}>\cdots>i_{1} \geq 1$ and $1 \leq j_{1}<\cdots<j_{r} \leq n$ such that $\gamma_{s}=\delta_{i_{s}}-\epsilon_{j_{s}}$, $s=1, \ldots, r$, are the atypical roots, namely, $\lambda_{m+1-i_{s}}^{\rho}=-\bar{\lambda}_{m+j_{s}}^{\rho}$ (recall notation below (3.1)). Following [36], we introduce the height vector of $\lambda^{(m \mid n)}$ :

$$
h\left(\lambda^{(m \mid n)}\right)=\left(h_{1}\left(\lambda^{(m \mid n)}\right), \ldots, h_{r}\left(\lambda^{(m \mid n)}\right)\right), \quad \text { with } h_{s}\left(\lambda^{(m \mid n)}\right)=\lambda_{m+1-i_{s}}-j_{s}+s .
$$

Now define a $\mathfrak{g l}_{r \mid r}$-weight $\lambda^{(r \mid r)} \in P_{0}^{+}(r \mid r)$ by

$$
\begin{aligned}
& \lambda^{(r \mid r)}+\rho^{(r \mid r)}=\left(h^{\prime}\left(\lambda^{(m \mid n)}\right) \mid-h\left(\lambda^{(m \mid n)}\right)\right), \quad \text { where } \\
& h^{\prime}\left(\lambda^{(m \mid n)}\right)=\left(h_{r}\left(\lambda^{(m \mid n)}\right), \ldots, h_{1}\left(\lambda^{(m \mid n)}\right)\right) .
\end{aligned}
$$

Note that we necessarily have $r \leq \min (m, n)$.

Remark 3.12. If we use weight diagrams to represent weights as in Section 5.1, the weight diagram of $\lambda^{(r \mid r)}$ is simply obtained from that of $\lambda^{(m \mid n)}$ by deleting all $>$ 's, <'s and their corresponding vertices, then re-indexing the remaining vertices.

The following result is due to Serganova [29] (see also [36, Remark 3.2]).

Theorem 3.13 ([29, 17]). Keep notation as above. There is an equivalence of categories $\mathcal{O}^{\mathfrak{p}_{m \mid n}}\left(\lambda^{(m \mid n)}\right) \stackrel{\sim}{\rightarrow} \mathcal{O}^{\mathfrak{p}_{r} \mid r}\left(\lambda^{(r \mid r)}\right)$, which in particular sends

$$
L^{m \mid n}\left(\mu^{(m \mid n)}\right) \rightarrow L^{r \mid r}\left(\mu^{(r \mid r)}\right), \quad K^{m \mid n}\left(\mu^{(m \mid n)}\right) \rightarrow K^{r \mid r}\left(\mu^{(r \mid r)}\right),
$$

for any $\mu^{(m \mid n)} \in P_{0}^{+}(m \mid n)$ belonging to the same block as $\lambda^{(m \mid n)}$, where $\mu^{(r \mid r)} \in P_{0}^{+}(r \mid r)$ is defined by (3.5).

Remark 3.14. This is a special case of Theorem 5.2 in [17], which also covers the orthosymplectic Lie superalgebras. The equivalence of categories sends Kac modules to Kac modules and irreducibles to irreducibles by [17, Lemma 7.14] and [29, Proposition 2.7]. Theorem 3.13 also follows from recent results of Brundan and Stroppel (see [9, IV, Theorem 1.1]).

Note that the category $\mathcal{O}^{\mathfrak{p}_{r \mid r}}\left(\lambda^{(r \mid r)}\right)$ of $\mathfrak{g l}_{r \mid r}$-modules is the maximally atypical block of $\mathcal{O}^{\mathfrak{p}_{r \mid r}}$ containing the trivial module.

As a corollary of Theorems 3.13 and 3.6, we have the following result on the Jantzen filtration for Kac modules. 
Lemma 3.15. Let $\lambda^{(m \mid n)}, \mu^{(m \mid n)} \in P_{0}^{+}(m \mid n)$ be in the same block. Denote by $\lambda^{(r \mid r)}$ and $\mu^{(r \mid r)}$ the corresponding weights in $P_{0}^{+}(r \mid r)$ defined by (3.5). Under the equivalence of categories of Theorem 3.13, the Jantzen filtration for $K^{(m \mid n)}\left(\lambda^{(m \mid n)}\right)$ corresponds to the Jantzen filtration for $K^{(r \mid r)}\left(\lambda^{(r \mid r)}\right)$. Furthermore, $J_{\lambda^{(m \mid n)} \mu^{(m \mid n)}}(q)=J_{\lambda^{(r \mid r)}} \mu^{(r \mid r)}(q)$.

Proof. The Jantzen filtration for $K^{(m \mid n)}\left(\lambda^{(m \mid n)}\right)$ is the radical filtration, which is sent to the radical filtration for $K^{(r \mid r)}\left(\lambda^{(r \mid r)}\right)$ by the equivalence of categories of Theorem 3.13. By Theorem 3.6, the radical filtration for $K^{(r \mid r)}\left(\lambda^{(r \mid r)}\right)$ is the Jantzen filtration. The second part of the lemma immediately follows.

\subsection{Statement 2.7 for $\mathfrak{g l}_{m \mid n}$}

Now we are in a position to prove the following theorem.

Theorem 3.16. Statement 2.7 holds for $\mathfrak{g l}_{m \mid n}$. That is, the decomposition numbers of the layers of the Jantzen filtration of any Kac module for $\mathfrak{g l}_{m \mid n}$ are determined by the coefficients of inverse Kazhdan-Lusztig polynomials.

Proof. The claim is true for typical Kac modules in a trivial way.

By Theorem 3.13 and Lemma 3.15, in order to show that the claim is true for $r$-fold atypical Kac modules for $\mathfrak{g l}_{m \mid N}$, it suffices to prove it for $r$-fold atypical Kac modules for $\mathfrak{g l}_{r \mid N}$ for any $N \geq r$.

Let $X_{r}^{+}$be the subset of $X^{+}(r \mid \infty)$ consisting of $r$-fold atypical weights. For any fixed element $\lambda \in X_{r}^{+}$, let $K(\lambda)=K^{(r \mid \infty)}(\lambda)$ and consider the Jantzen filtration for $K(\lambda)$,

$$
K(\lambda)=K^{0}(\lambda) \supset K^{1}(\lambda) \supset \cdots \supset K^{r}(\lambda) \supset\{0\},
$$

which is defined to be the direct limit of the Jantzen filtrations of $K^{(r \mid N)}(\lambda), N \geq r$ (that is, each $K^{i}(\lambda)$ is a direct limit). Since by Theorem 3.6 the Jantzen filtration for every finite $N$ is a radical filtration, (3.6) is also a radical filtration with the consecutive quotients $K_{i}(\lambda)=K^{i}(\lambda) / K^{i+1}(\lambda)$ being semisimple. Let

$$
\Sigma_{r}^{i}(\lambda)=\left\{\mu \in X_{r}^{+} \mid\left[K_{i}(\lambda): L^{(r \mid \infty)}(\mu)\right]>0\right\}, \quad \text { which is a finite set. }
$$

Set $\Sigma_{r}(\lambda)=\bigcup_{i=0}^{r} \Sigma_{r}^{i}(\lambda)$. Since the multiplicity of each composition factor of $K(\lambda)$ is at most $1, \Sigma_{r}^{i}(\lambda) \cap \Sigma_{r}^{j}(\lambda)=\{0\}$ if $i \neq j$. Furthermore, we may replace the condition $\left[K_{i}(\lambda): L^{(r \mid \infty)}(\mu)\right]>0$ by $\left[K_{i}(\lambda): L^{(r \mid \infty)}(\mu)\right]=1$ in the definition of $\Sigma_{r}^{i}$.

The super duality functor of Theorem 3.10 sends (3.6) to a filtration

$$
M\left(\lambda^{\natural}\right)=M^{0}\left(\lambda^{\natural}\right) \supset M^{1}\left(\lambda^{\natural}\right) \supset \cdots \supset M^{r}\left(\lambda^{\natural}\right) \supset\{0\}
$$

of the generalised Verma module $M\left(\lambda^{\natural}\right)=M^{(r+\infty)}\left(\lambda^{\natural}\right)$ of $\mathfrak{g l}_{r+\infty}$. It is crucial to observe that (3.7) is a radical filtration since (3.6) is. The semisimple consecutive quotients $M_{i}\left(\lambda^{\natural}\right)=M^{i}\left(\lambda^{\natural}\right) / M^{i+1}\left(\lambda^{\natural}\right)$ of (3.7) satisfy

$$
\left[M_{i}\left(\lambda^{\natural}\right): L^{(r+\infty)}(\mu)\right]= \begin{cases}1 & \text { if } \mu^{\natural} \in \Sigma_{r}^{i}(\lambda), \\ 0 & \text { otherwise. }\end{cases}
$$


Since $\Sigma_{r}(\lambda)$ is a finite set, there exists an $n_{0}$ such that for any $N \geq n_{0}$, we have the equalities of multiplicities

$$
\begin{aligned}
& {\left[\operatorname{tr}_{N} K_{i}(\lambda): L^{(r \mid N)}(\mu)\right]=\left[K_{i}(\lambda): L^{(r \mid \infty)}(\mu)\right],} \\
& {\left[\operatorname{tr}_{N} M_{i}\left(\lambda^{\natural}\right): L^{(r+N)}\left(\mu^{\natural}\right)\right]=\left[M_{i}\left(\lambda^{\natural}\right): L^{(r+\infty)}(\mu)\right]}
\end{aligned}
$$

for all $i$ and $\mu \in \Sigma_{r}(\lambda)$.

The exact truncation functor $\operatorname{tr}_{N}$ maps the radical filtration (3.7) for $M\left(\lambda^{\natural}\right)$ to a radical filtration

$$
\operatorname{tr}_{N} M^{0}\left(\lambda^{\natural}\right) \supset \operatorname{tr}_{N} M^{1}\left(\lambda^{\natural}\right) \supset \cdots \supset \operatorname{tr}_{N} M^{r}\left(\lambda^{\natural}\right) \supset\{0\}
$$

for the $\mathfrak{g l}_{r+N}$-module $\operatorname{tr}_{N} M\left(\lambda^{\natural}\right)$. Note that $\operatorname{tr}_{N} M\left(\lambda^{\natural}\right)$ is the generalised Verma module $M^{(r+N)}\left(\lambda^{\natural}\right)$, and $\operatorname{tr}_{N} M_{i}\left(\lambda^{\natural}\right)=\operatorname{tr}_{N} M^{i}\left(\lambda^{\natural}\right) / \operatorname{tr}_{N} M^{i+1}\left(\lambda^{\natural}\right)$ for all $i$. It is clear that

$$
\begin{aligned}
J_{\lambda \mu}(q) & :=\sum_{i=0}^{r} q^{i}\left[\operatorname{tr}_{N} K_{i}(\lambda): L^{(r \mid N)}(\mu)\right]=\sum_{i=0}^{r} q^{i}\left[K_{i}(\lambda): L^{(r \mid \infty)}(\mu)\right] \\
& =\sum_{i=0}^{r} q^{i}\left[\operatorname{tr}_{N} M_{i}\left(\lambda^{\natural}\right): L^{(r+N)}\left(\mu^{\natural}\right)\right] .
\end{aligned}
$$

By $[15,6,21], \mathcal{O}_{\text {int }}^{\mathfrak{p}_{m+N}}$ is a multiplicity free highest weight category with rigid generalised Verma modules. Furthermore, the layers of the radical filtration of a generalized Verma module are described by the coefficients of inverse Kazhdan-Lusztig polynomials [21, Corollary 7.1.3] (see also [6]). Therefore, for the generalized Verma module $M^{(r+N)}\left(\lambda^{\natural}\right)$ in $\mathcal{O}_{\text {int }}^{\mathfrak{p}_{m+N}}$,

$$
\sum_{i=0}^{r} q^{i}\left[\operatorname{tr}_{N} M_{i}\left(\lambda^{\natural}\right): L^{(r+N)}\left(\mu^{\natural}\right)\right]=b_{\lambda^{\natural} \mu^{\natural}}^{(r+N)}(q) .
$$

Remark 3.17. This formula follows from [21, Corollary 7.1.3] upon converting conventions for Kazhdan-Lusztig polynomials. The author of [21] himself found out that some proofs in the paper had flaws, which, however, could all be rectified. The results of the paper remain valid.

For $N$ sufficiently large, we have $b_{\lambda^{\natural} \mu^{\natural}}^{(r+N)}(q)=a_{\lambda \mu}^{(r \mid N)}(q)$. This immediately leads to $J_{\lambda \mu}(q)=a_{\lambda \mu}^{(r \mid N)}(q)$. Using the second part of Lemma 3.15, we conclude that Statement 2.7 holds for any $r$-fold atypical block in $\mathcal{O}_{\text {int }}^{\mathfrak{p}_{m} \mid n}(r \leq \min (m, n))$. Since every Kac module can be turned into an object of $\mathcal{O}_{\text {int }}^{\mathfrak{p}_{m} \mid n}$ by tensoring with an appropriate 1-dimensional module, Statement 2.7 also holds in $\mathcal{O}^{\mathfrak{p}_{m} \mid n}$ by the first part of Lemma 3.5. This completes the proof of the theorem.

Remark 3.18. By using methods of Section 5 and techniques from [36], one can prove Theorem 3.16 without resorting to "super duality". 


\section{Proof of Lemma 3.7}

In this section we prove Lemma 3.7, which was used in the proof of Theorem 3.6. To do this, we need to introduce some notions related to submodule lattices of Kac modules. We continue to denote $\mathfrak{g l}_{m \mid n}$ by $\mathfrak{g}$ throughout the section.

\subsection{Primitive weight graphs}

For a primitive weight $\mu$ of a $\mathfrak{g}$-module $V$, we shall use $v_{\mu}$ to denote a nonzero primitive vector of weight $\mu$ which generates an indecomposable submodule. Two primitive vectors which generate the same indecomposable submodule are regarded the same. Denote by $P(V)$ the multi-set of primitive weights of $V$, where the multiplicity $m_{\mu}$ of a primitive weight $\mu$ is equal to the dimension of the subspace spanned by all the primitive vectors with weight $\mu$. In the case when $V$ is a submodule or subquotient of a Kac module for $\mathfrak{g}$, all primitive weights have multiplicity 1 . For $\mu, v \in P(V)$, if $\mu \neq v$ and $v_{v} \in \mathrm{U}(\mathfrak{g}) v_{\mu}$, we say that $\nu$ is derived from $\mu$ and write $v \leftarrow \cdots-\mu$ or $\mu-\cdots \rightarrow v$. If $\mu \rightarrow \cdots \rightarrow v$ and there exists no $\lambda \in P(V)$ such that $\mu \rightarrow \cdots \rightarrow \lambda \rightarrow \cdots \rightarrow \nu$, then we say that $\nu$ is directly derived from $\mu$ and write $\mu \rightarrow v$ or $v \leftarrow \mu$. Occasionally we use $\mu \stackrel{e}{\leftarrow}-v, v \stackrel{e}{\leftarrow}-\mu, \mu \stackrel{e}{\leftarrow} v, v \stackrel{e}{\rightarrow} \mu$ to emphasise the fact that $v_{\mu} \in \mathrm{U}\left(\mathfrak{g}^{+}\right) \mathfrak{g}^{+} v_{\nu}$.

Definition 4.1. We associate with $P(V)$ a directed graph, still denoted by $P(V)$, in the following way: the vertices of the graph are elements of the multi-set $P(V)$. Two vertices $\lambda$ and $\mu$ are connected by a single directed edge pointing toward $\mu$ if and only if $\mu$ is derived from $\lambda$. We shall call this graph the primitive weight graph of $V$. The skeleton of the primitive weight graph is the subgraph containing all the vertices of $P(V)$ such that two vertices $\lambda$ and $\mu$ are connected (by a single directed edge pointing toward $\mu$ ) if and only if $\mu$ is directly derived from $\lambda$ (in this case we say that the two weights are linked).

Note that a primitive weight graph is uniquely determined by its skeleton.

A full subgraph $S$ of $P(V)$ is a subset of $P(V)$ which contains all the edges linking vertices of $S$. We call a full subgraph $S$ closed if $\mu-\cdots \rightarrow \eta-\cdots \rightarrow v$ implies $\eta \in S$ for any $\eta \in P(V)$ and $\mu, v \in S$. It is clear that a module is indecomposable if and only if its primitive weight graph is connected (in the usual sense), and that a full subgraph of $P(V)$ corresponds to a subquotient of $V$ if and only if it is closed. Thus a full subgraph with only two weights is always closed. For a directed graph $\Gamma$, we denote by $M(\Gamma)$ any module with primitive weight graph $\Gamma$ if such a module exists. If $\Gamma$ is a closed full subgraph of $P(V)$, then $M(\Gamma)$ always exists, which is a subquotient of $V$.

Definition 4.2. A subgraph of $P(V)$ of the form $\mu_{0}-\cdots \rightarrow \mu_{1}-\cdots \rightarrow \cdots-\cdots \rightarrow \mu_{k}$ is called a chain of length $k$. If $\mu_{i+1}$ is directly derived from $\mu_{i}$ for every $i$, we say that the chain is exact.

Remark 4.3. If every composition factor of $V$ is a highest weight module, the primitive weight graph $P(V)$ provides a convenient graphical representation of the submodule lattice $S(V)$ of $V$. A chain in $P(V)$ corresponds to a chain in $S(V)$ with the submodules 
being those generated by the primitive vectors. If a chain in $P(V)$ is exact, the corresponding chain in $S(V)$ has the property that every inclusion of a submodule by a neighbour in the chain is a covering.

Remark 4.4. Note the difference in the terminologies used here and in [35]. In the terminology of this paper, [35, Definition 6.2] was for the skeleton of a primitive weight graph.

Since the Jantzen filtration for the Kac module $K(\lambda)$ has semisimple consecutive quotients (see the first part in the proof of Theorem 3.6), and has length $r=\sharp(\lambda)$ (by Theorem 3.2), one immediately obtains the following result.

Corollary 4.5. Every chain in the primitive weight graph $P(K(\lambda))$ of the Kac module $K(\lambda)$ has length at most $r=\sharp(\lambda)$.

Proof. The existence of a longer chain would imply that some of the consecutive quotients of the Jantzen filtration for $K(\lambda)$ were not semisimple.

\subsection{Proof of Lemma 3.7}

We can reformulate Lemma 3.7 as follows.

Lemma 4.6. The primitive weight graph $P(K(\lambda))$ of the Kac module $K(\lambda)$ contains at least one chain of length $r=\sharp(\lambda)$.

It is this reformulation of Lemma 3.7 which we shall prove below by constructing a chain of length $r$ in the primitive weight graph.

Theorem 3.13 reduces the task at hand to the case where $\mathfrak{g}=\mathfrak{g l}_{r \mid r}$ and $\lambda$ is $r$-fold atypical. For $1 \leq k \leq r$, let $\mathfrak{g}_{[1, k]}$ be the subalgebra of $\mathfrak{g}$ spanned by $e_{a b}$ with $r-k<$ $a, b \leq r+k$. Choose the standard triangular decomposition for $\mathfrak{g}_{[1, k]}$ and denote by $\Delta_{k}$ the set of roots. Let $\mathfrak{g}_{[1, k]}^{+}$be the strictly upper triangular subalgebra of $\mathfrak{g}_{[1, k]}$, that is, the nilpotent radical of the standard Borel subalgebra. We similarly denote by $\mathfrak{g}_{[1, k]}^{-}$the strictly lower triangular subalgebra of $\mathfrak{g}_{[1, k]}$. The weight $\lambda$ restricted to $\mathfrak{g}_{[1, k]}$ is denoted by $\lambda_{[1, k]}$.

Let $v_{\lambda}$ be the highest weight vector that generates the Kac module $K(\lambda)$ for $\mathfrak{g}$, and set $v_{0}=v_{\lambda}$. Regard $K(\lambda)$ as a module over $\mathfrak{g}_{[1, k]}$, and let $V_{k}=\mathrm{U}\left(\mathfrak{g}_{[1, k]}\right) v_{\lambda}$ be the $\mathfrak{g}_{[1, k]^{-}}$ submodule of $K(\lambda)$ generated by $v_{\lambda}$. Obviously, $V_{k}$ is isomorphic to the Kac module $K\left(\lambda_{[1, k]}\right)$ for $\mathfrak{g}_{[1, k]}$. We also have the inclusions $V_{1} \subset \cdots \subset V_{r}$. The primitive vector $v_{k}$ of the socle (that is, the bottom composition factor) of the $\mathfrak{g}_{[1, k]}$-submodule $V_{k}$ is strongly $\mathfrak{g}_{[1, k]}$-primitive, namely, $\mathfrak{g}_{[1, k]}^{+} v_{k}=\{0\}$. Furthermore, $v_{k} \in \mathrm{U}\left(\mathfrak{g}_{[1, k]}^{-}\right) v_{\lambda}$. Since every simple root vector $X_{\alpha} \in \mathfrak{g}$ associated with a simple root $\alpha \notin \Delta_{k}$ commutes with $\mathfrak{g}_{[1, k]}^{-}$, we have $X_{\alpha} v_{k}=0$ for all simple roots $\alpha$ of $\mathfrak{g}$, i.e., $v_{k}$ is a strongly $\mathfrak{g}$-primitive vector for $k=1, \ldots, r$.

Denote the $\mathfrak{g}$-weight of $v_{k}$ by $\lambda_{k}$ for $k=0,1, \ldots, r$, where $\lambda_{0}=\lambda$. All $\lambda_{k}$ can be worked out by using, e.g., [39, Proposition 3.6.]. In the terminology of $[18,33], \lambda_{k}$ corresponds to the boundary strip removals of the first $k$ atypical roots of the composite 
Young diagram of $\lambda$ since $\lambda$ is $r$-fold atypical. It is related to $\lambda$ by $\lambda=R_{\theta^{k}}\left(\lambda_{k}\right)$, where $R_{\theta^{k}}$ is the raising operator of Definition 5.1, and $\theta^{k}=(\underbrace{1, \ldots, 1}_{k}, \underbrace{0, \ldots, 0}_{r-k})$. We have $\lambda\left(e_{r-k, r-k}\right)=\lambda_{k}\left(e_{r-k, r-k}\right)>\lambda_{k+1}\left(e_{r-k, r-k}\right)$ for each $k$, hence $\lambda_{i} \neq \lambda_{j}$ if $i \neq j$.

Now we consider $v_{k-1} \in V_{k-1} \subset V_{k}$. Being a strongly $\mathfrak{g}_{[1, k]}$-primitive vector, $v_{k-1}$ generates a $\mathfrak{g}_{[1, k]}$-submodule in $V_{k}$ that contains the bottom composition factor of $V_{k}$. Since $v_{k}$ is the $\mathfrak{g}_{[1, k]}$-primitive vector of the bottom composition factor of $V_{k} \cong K\left(\lambda_{[1, k]}\right)$, obviously $v_{k}$ is generated by $v_{k-1}$ as $\mathfrak{g}_{[1, k]}$-primitive vectors. We have already shown that $v_{j}$ is strongly $\mathfrak{g}$-primitive for all $j$, thus in the primitive weight graph $P(K(\lambda))$ of the Kac module $K(\lambda)$ for $\mathfrak{g}$, there exists a chain

$$
\lambda_{0}-\cdots \rightarrow \lambda_{1}-\cdots \rightarrow \cdots-\cdots \rightarrow \lambda_{r} .
$$

This proves Lemma 4.6, which is equivalent to Lemma 3.7.

Remark 4.7. By Corollary 4.5, the longest possible length of any chain in $P(K(\lambda))$ is $r=\sharp(\lambda)$. This forces the chain (4.1) to be exact. Thus, we have constructed an exact chain

$$
\lambda_{0} \rightarrow \lambda_{1} \rightarrow \cdots \rightarrow \lambda_{r}
$$

of length $r$ in the primitive weight graph $P(K(\lambda))$ of the Kac module $K(\lambda)$.

\section{Submodule lattices of Kac modules}

In this section we utilise knowledge of the Jantzen filtration to study the structure of Kac modules. The main result obtained is Theorem 5.18, which describes the chains in the submodule lattice of each Kac module. The theorem is stated in terms of the primitive weight graph, a graphical representation of the submodule lattice discussed in the last section (see Remark 4.3). As already alluded to in Section 1, Theorem 5.18 is part of unpublished conjectures of Hughes, King and van der Jeugt [19].

\subsection{Left and right moves on weight diagrams}

In this subsection we describe certain combinatorial operations on weight diagrams [9] (see also [26, 17]), which will play an important role in the remainder of the paper.

Hereafter we adopt a new convention for the coordinates of weights relative to the basis $\left\{\delta_{i} \mid 1 \leq i \leq m\right\} \cup\left\{\epsilon_{j} \mid 1 \leq j \leq n\right\}$ of $\mathfrak{h}^{*}$ described at the beginning of Section 3.2. A weight $\lambda \in \mathfrak{h}^{*}$ will be written as

$$
\lambda=\left(\lambda_{m}, \ldots, \lambda_{1} \mid \dot{\lambda}_{1}, \ldots, \dot{\lambda}_{n}\right)=\sum_{a=1}^{m} \lambda_{a} \delta_{a}-\sum_{b=1}^{n} \dot{\lambda}_{b} \epsilon_{b} .
$$

We also use the notation

$$
\lambda^{\rho}=\lambda+\rho=\left(\lambda_{m}^{\rho}, \ldots, \lambda_{1}^{\rho} \mid \dot{\lambda}_{1}^{\rho}, \ldots, \dot{\lambda}_{n}^{\rho}\right),
$$


where $\lambda_{a}^{\rho}=\lambda_{a}+a-1$ and $\dot{\lambda}_{b}^{\rho}=\dot{\lambda}_{b}+b-1$. Denote

$$
\begin{aligned}
S(\lambda)_{\mathrm{L}} & =\left\{\lambda_{a}^{\rho} \mid a=1, \ldots, m\right\}, & & S(\lambda)_{\mathrm{R}}=\left\{\dot{\lambda}_{b}^{\rho} \mid b=1, \ldots, n\right\}, \\
S(\lambda) & =S(\lambda)_{\mathrm{L}} \cup S(\lambda)_{\mathrm{R}}, & & S(\lambda)_{\mathrm{B}}=S(\lambda)_{\mathrm{L}} \cap S(\lambda)_{\mathrm{R}} .
\end{aligned}
$$

Following $[9,17]$, one can represent every integral element $\lambda$ of $P_{0}^{+}$in a unique way by a weight diagram $D_{\lambda}$, which is a line with vertices indexed by $\mathbb{Z}$ such that vertex $i$ is associated with a symbol $D_{\lambda}^{i}=\emptyset,<,>$ or $\times$ according to whether $i \notin S(\lambda), i \in$ $S(\lambda)_{\mathrm{R}} \backslash S(\lambda)_{\mathrm{B}}, i \in S(\Lambda)_{\mathrm{L}} \backslash S(\lambda)_{\mathrm{B}}$ or $i \in S(\lambda)_{\mathrm{B}}$. Thus the degree $\sharp(\lambda)$ of atypicality of $\lambda$ is the number of $\times$ 's in the weight diagram $D_{\lambda}$.

For example, If $\lambda^{\rho}=(7,5,4,2,1 \mid 1,2,4,7,8,10)$, the weight diagram is given by

$$
\cdots-0-\stackrel{\times}{1}-\stackrel{\times}{2}-3-\stackrel{\times}{4}-\stackrel{>}{5}-6-\stackrel{\times}{7}-\stackrel{<}{8}-9-\stackrel{<}{-10}-11-\cdots
$$

where, for simplicity, we have left vertex $i$ unmarked if $D_{\lambda}^{i}=\emptyset$. Note that $\sharp(\lambda)=4$, which is the number of $x$ 's in (5.3).

Given a weight diagram $D_{\lambda}$, we define $\ell_{\lambda}(s, t)$ to be the number of $\times$ 's minus the number of $\emptyset$ 's strictly between vertices $s$ and $t$. Suppose $\sharp(\lambda)=r$. We label the $\times$ 's in $D_{\lambda}$ by $1, \ldots, r$ from left to right, and denote the vertex where the $i$-th $\times$ sits by $x_{i}$. A right move (or raising operator) on $D_{\lambda}$ is to move to the right a $\times$, say the $i$-th one, to the first empty vertex $t$ (vertex with the symbol $\emptyset$ ) that meets the conditions $\ell_{\lambda}\left(x_{i}, t\right)=0$ and $\ell_{\lambda}\left(x_{i}, s\right)>0$ for all vertices $s$ satisfying $x_{i}<s<t$. We denote this right move by $R_{i}(\lambda)$. Note that the condition $\ell_{\lambda}\left(x_{i}, t\right)=0$ forces the numbers of $\times$ 's and $\emptyset$ 's strictly between $x_{i}$ and $t$ to be equal. If this number is $k$, we let $j=i+k$ and also denote $R_{i}(\lambda)$ by $R_{i, j}(\lambda)$. As an example, we observe that the first $\times$ in the weight diagram (5.3) can only be moved to vertex 11 , which is the move $R_{1}(\lambda)$ or $R_{1,4}(\lambda)$.

A left move (or lowering operator) is to move to the left a $\times$, say the $j$-th one, to any empty vertex $s$ such that $\ell_{\lambda}\left(s, x_{j}\right)=0$. If the number of $\times$ 's strictly between $s$ and $x_{j}$ is $k$, we let $i=j-k$ and denote the left move by $L_{i, j}(\lambda)$.

Note that a left move may move a $\times$ to any empty vertex on its left so long as it passes the same number of $\times$ 's and $\emptyset$ 's, in contrast to a right move.

Definition 5.1. Given an element $\theta=\left(\theta_{1}, \ldots, \theta_{r}\right) \in\{0,1\}^{r}$, we set $|\theta|=\sum_{i=1}^{r} \theta_{i}$ and let $\theta_{i_{1}}, \ldots, \theta_{i_{|\theta|}}$ with $1 \leq i_{1}<\cdots<i_{|\theta|} \leq r$ be the nonzero entries. Associate to $\theta$ a unique right path $R_{\theta}(\lambda)$ which is the collection of the $|\theta|$ right moves $R_{i_{1}}(\lambda), \ldots, R_{i_{|\theta|}}(\lambda)$. We also use $R_{\theta}(\lambda)$ to denote the integral dominant weight corresponding to the weight diagram obtained in the following way. For each $a=1, \ldots,|\theta|$, let $t_{a}$ be the vertex to which the $i_{a}$-th $\times$ of $D_{\lambda}$ is moved by $R_{i_{a}}(\lambda)$. Delete from $D_{\lambda}$ all the $\times$ 's labelled by $i_{1}, \ldots, i_{|\theta|}$, and then place $\mathrm{a} \times$ at each of the vertices $t_{1}, \ldots, t_{|\theta|}$.

Definition 5.2. A left path (or simply a path) is the collection of left moves $L_{i_{1}, j_{1}}(\lambda), \ldots$, $L_{i_{k}, j_{k}}(\lambda)$ satisfying all of the following conditions:

(1) $1 \leq j_{1}<\cdots<j_{k} \leq r$;

(2) for $1 \leq a<b \leq k$, if $i_{b} \leq j_{a}$ then $i_{b} \leq i_{a}$;

(3) for any $i_{b} \leq p<j_{b}$, if $\ell_{\lambda}\left(x_{p}, x_{j_{b}}\right) \geq 0$, then $p=j_{a}$ for some $a<b$. 
Let $\boldsymbol{i}=\left(i_{1}, \ldots, i_{k}\right)$ and $\boldsymbol{j}=\left(j_{1}, \ldots, j_{k}\right)$, and denote by $L_{\boldsymbol{i}, \boldsymbol{j}}(\lambda)$ the left path. If $k=0$, we use $L_{\emptyset}$ to denote this empty path. We shall also use $L_{i, j}(\lambda)$ to denote the integral dominant weight corresponding to the weight diagram obtained in the following way. Let $s_{a}$ be the vertex to which the $j_{a}$-th $\times$ of $\lambda$ is moved by $L_{i_{a}, j_{a}}(\lambda)$ for $a=1, \ldots, k$. Delete from $\lambda$ the $\times$ 's labelled by $j_{1}, \ldots, j_{k}$ and then place a $\times$ at each of the vertices $s_{1}, \ldots, s_{k}$.

Remark 5.3. We put $\lambda$ in the notations $R_{i_{a}}(\lambda)$ and $L_{i_{b}, j_{b}}(\lambda)$ to emphasise the fact that the individual moves in a left path $L_{i, j}(\lambda)$ or right path $R_{\theta}(\lambda)$ are independently applied to the weight diagram $D_{\lambda}$ of $\lambda$, and not to the diagram resulting from previous moves.

Remark 5.4. In the language developed here, [28, Theorem 5.5] and [9, IV, Lemma 2.11] state that for a dominant weight $\lambda$ satisfying the given conditions, $L(\mu)$ is a composition factor of $K(\lambda)$ if $\mu$ is obtained from $\lambda$ by a single left move.

Remark 5.5. By [8, Main Theorem] (also see [39, Conjecture 4]),

$$
\mu \in P(K(\lambda)) \quad \text { iff } \quad \lambda=R_{\theta}(\mu) \text { for some } \theta \in\{0,1\}^{r} .
$$

Also the set $P(K(\lambda))$ of primitive weights of $K(\lambda)$ is exactly the set of integral dominant weights corresponding to paths (i.e., left paths) [33].

Example 5.6. If $\lambda$ is the weight in (5.3), one can easily obtain all the possible left paths for $D_{\lambda}$. There are 19 paths in total, which are given by

$$
\begin{array}{lllll}
L_{\emptyset} & L_{11} & L_{11} L_{12} & L_{33} & L_{11} L_{33} \\
L_{11} L_{12} L_{33} & L_{11} L_{13} & L_{44} & L_{11} L_{44} & L_{11} L_{12} L_{44} \\
L_{33} L_{44} & L_{11} L_{33} L_{44} & L_{11} L_{12} L_{33} L_{44} & L_{11} L_{13} L_{44} & L_{34} \\
L_{11} L_{34} & L_{11} L_{12} L_{34} & L_{11} L_{14} & L_{11} L_{33} L_{14} &
\end{array}
$$

When we work with a fixed weight $\lambda$ and there is no possibility of confusion, we drop $\lambda$ from the notation for left and right moves.

Given a left path $L_{\boldsymbol{i}, \boldsymbol{j}}(\lambda)$, let $i_{0}=\min \left\{i_{1}, \ldots, i_{k}\right\}$. Then the length $\ell\left(L_{\boldsymbol{i}, \boldsymbol{j}}(\lambda)\right)$, range $r\left(L_{\boldsymbol{i}, \boldsymbol{j}}(\lambda)\right)$ and depth $d\left(L_{\boldsymbol{i}, \boldsymbol{j}}(\lambda)\right)$ of the path are respectively defined to be $k,\left[i_{0}, j_{k}\right]$ and $j_{k}-i_{0}$, where we have used the notation $[i, j]=\{i, i+1, \ldots, j\}$.

Any subsequence of the left path $L_{i, j}(\lambda)$ is called a subpath of $L_{i, j}(\lambda)$ if it forms itself a left path. Thus the subsequence of left moves $L_{i_{1}, j_{1}}(\lambda), L_{i_{2}, j_{2}}(\lambda), \ldots, L_{i_{a}, j_{a}}(\lambda)$ is a subpath of $L_{i, j}(\lambda)$ for any $1 \leq a \leq k$.

Two left paths $L_{\boldsymbol{i}, \boldsymbol{j}}(\lambda), L_{\boldsymbol{i}^{\prime}, \boldsymbol{j}^{\prime}}(\lambda)$ are disjoint if $j_{k}<i_{a}^{\prime}$ for all $a$. In this case, putting two paths together, we obtain a path $L_{\boldsymbol{i}^{\prime \prime}} \boldsymbol{j}^{\prime \prime}(\lambda)$, where $\boldsymbol{i}^{\prime \prime}=\left(i_{1}, \ldots, i_{k}, i_{1}^{\prime}, \ldots, i_{k^{\prime}}^{\prime}\right), \boldsymbol{j}^{\prime \prime}=$ $\left(j_{1}, \ldots, j_{k}, j_{1}^{\prime}, \ldots, j_{k^{\prime}}^{\prime}\right)$. We denote this path by $L_{i, j}(\lambda) L_{\boldsymbol{i}^{\prime} \boldsymbol{j}^{\prime}}(\lambda)$ and call it the disjoint sum of the paths $L_{i, j}(\lambda)$ and $L_{\boldsymbol{i}^{\prime} \boldsymbol{j}^{\prime}}(\lambda)$.

Call a left path $L_{i, j}(\lambda)$ indecomposable if $i_{k} \leq i_{1}$. Then every left path can be uniquely decomposed as a disjoint sum of indecomposable subpaths; each indecomposable component is called a block of the path.

A left path is called a bridge or a path with bridges if for some $a, b$ with $i_{a} \leq b<j_{a}$, the $b$-th $\times$ is not moved in the path, i.e., $b \neq j_{c}$ for any $c$. 
Remark 5.7. (1) Among the paths on $D_{\lambda}$, there is a unique one of length $r=\sharp(\lambda)$, called the bottom path and denoted $L_{B}$, which corresponds to the bottom composition factor of $K(\lambda)$. The third path in the third row of (5.5) is the bottom path.

(2) For each $0 \leq k \leq r$, there is a unique path $L_{[1, k]}$ on $D_{\lambda}$ without bridges, which moves all of the first $k \times$ 's. For example, in (5.6), the $L_{[1, k]}$ 's are: $L_{\emptyset}, L_{11}, L_{11} L_{12}$, $L_{11} L_{12} L_{33}, L_{11} L_{12} L_{33} L_{44}$. Obviously, $L_{[1, k]}$ is a subpath of $L_{[1, k+1]}$.

(3) An indecomposable path is a path without bridges if and only if its length equals its depth.

Remark 5.8. There is a one-to-one correspondence between paths and permissible codes defined in [18]. Paths without bridges correspond to unlinked codes; the corresponding primitive vectors are strongly primitive and have been constructed in [34]. For example, the codes corresponding to the paths in (5.5) are

$\begin{array}{lllll}0000 & 1000 & 1200 & 0030 & 1030 \\ 1230 & 1330 & 0004 & 1004 & 1204 \\ 2034 & 1034 & 1234 & 1334 & 0044 \\ 1044 & 1244 & 14444 & 14434 & \end{array}$

where codes with the same nonzero labels in the first row correspond to linked codes.

Remark 5.9. Weight diagrams provide a convenient combinatorial tool for studying representations of Lie superalgebras. The equivalence of the two algorithms (respectively developed in [28] and [8]) for computing the composition factors and multiplicities of Kac modules for $\mathfrak{g l}_{m \mid n}$ was proven in [26] with the help of weight diagrams.

Remark 5.10. The coefficient of $q^{k}$ in the generalised Kazhdan-Lusztig polynomial $p_{\lambda \mu}(-q)$ is expected to be equal to the number of all regular decreasing paths (defined in [17, §13]) from $\mu$ to $\lambda$ of length $k$. We can prove this if $\lambda=R_{\theta}^{\prime}(\mu)$ for some $\theta=\left(\theta_{1}, \ldots, \theta_{\sharp \lambda}\right)$ satisfying $\theta_{i} \leq 1$ for all $i$, where $R_{\theta}^{\prime}$ is the raising operator defined by $[36,(3.32)]$. It will be very interesting to prove this in general.

\subsection{Technical lemmas}

We shall investigate the structures of the Kac modules $K(\lambda)$. Choose a basis $B$ of $\mathrm{U}\left(\mathfrak{g}_{-1}\right)$ : $B=\left\{b=\prod_{\beta \in S} X_{-\beta} \mid S \subset \Delta_{1}^{+}\right\}$, where the product $\prod_{\beta \in S} X_{-\beta}=X_{-\beta_{1}} \cdots X_{-\beta_{s}}$ is written in the proper order: $\beta_{1}<\cdots<\beta_{s}$ and $s=|S|$ (the level of $b$ ). Define a total order on $B$ :

$$
b>b^{\prime}=X_{-\beta_{1}^{\prime}} \cdots X_{-\beta_{s^{\prime}}^{\prime}} \Leftrightarrow s>s^{\prime} \text { or } s=s^{\prime}, \beta_{k}>\beta_{k}^{\prime}, \beta_{i}=\beta_{i}^{\prime}(1 \leq i \leq k-1),
$$

where $b, b^{\prime}$ are in proper order. Recall that an element $v \in K(\lambda)$ can be uniquely written as

$$
v=b_{1} y_{1} v_{\lambda}+\cdots+b_{t} y_{t} v_{\lambda}, \quad b_{i} \in B, b_{1}>\cdots>b_{t}, 0 \neq y_{i} \in \mathrm{U}\left(\mathfrak{g}_{0}^{-}\right) .
$$


Clearly $v=0 \Leftrightarrow t=0$. If $v \neq 0$, we call $b_{1} y_{1} v_{\lambda}$ the leading term. A term $b_{i} y_{i} v_{\lambda}$ is called a prime term if $y_{i} \in \mathbb{C}$; in this case $b_{i}$ is called a prime coefficient. Note that a vector $v$ may have zero or more than one prime terms.

Denote by $\bar{\lambda}$ the lowest weight in $L^{0}(\lambda)$, which is given by

$$
\bar{\lambda}=\left(\lambda_{1}, \ldots, \lambda_{m} \mid \dot{\lambda}_{n}, \ldots, \dot{\lambda}_{1}\right) \text {. }
$$

Denote $\bar{v}_{\lambda}$ the lowest weight vector in $L^{0}(\lambda)$. Similar to (5.7), a vector $v \in K(\lambda)$ can be uniquely written as

$$
v=b_{1} y_{1} \bar{v}_{\lambda}+\cdots+b_{t} y_{t} \bar{v}_{\lambda}, \quad b_{i} \in B, b_{1}<\cdots<b_{t}, 0 \neq y_{i} \in \mathrm{U}\left(\mathfrak{g}_{0}^{+}\right) .
$$

We can similarly define the lowest leading term, lowest prime terms, lowest prime coefficients. Similar to $\mathfrak{g}_{0}$-highest weight primitive vectors, a $\mathfrak{g}_{0}$-lowest weight vector $v$ in $K(\lambda)$ is primitive if $v$ generates an indecomposable $\mathfrak{g}$-submodule and there exists a $\mathfrak{g}$ submodule $W$ of $V$ such that $v \notin W$ but $\mathfrak{g}_{-1} v \in W$.

One immediately has [34]

Lemma 5.11. (1) Let $v=g u, u \in K(\lambda), g \in \mathrm{U}\left(\mathfrak{g}^{-}\right)$. If $u$ has no prime term then $v$ has no prime term.

(2) Let $v^{\prime}=g u^{\prime}$ with $u^{\prime} \in K(\lambda)$. If $u$, $u^{\prime}$ have the same prime terms then $v, v^{\prime}$ have the same prime terms.

(3) Let $v_{\mu} \in K(\lambda)$ be a $\mathfrak{g}_{0}$-highest vector with weight $\mu$. Then $\lambda-\mu$ is a sum of distinct positive odd roots, furthermore the leading term $b_{1} y_{1} v_{\lambda}$ of $v_{\mu}$ must be a prime term.

(4) Suppose $v_{\mu}^{\prime}=\sum_{i=1}^{t^{\prime}}\left(b_{i}^{\prime} y_{i}^{\prime}\right) v_{\lambda}$ is another $\mathfrak{g}_{0}$-highest vector with weight $\mu$. If all prime terms of $v_{\mu}$ are the same as those of $v_{\mu}^{\prime}$, then $v_{\mu}=v_{\mu}^{\prime}$.

Although our arguments below work perfectly well for any $r$-fold atypical weight $\lambda$ of $\mathfrak{g l}_{m \mid n}$, we restrict ourselves to the case $\mathfrak{g}=\mathfrak{g l}_{r \mid r}$ to simplify matters. Thanks to Theorem 3.13 , this will not lead to any loss of generality. In this case, an $r$-fold atypical weight $\lambda$ has the form $\lambda=\left(\lambda_{r}, \ldots, \lambda_{1} \mid \lambda_{1}, \ldots, \lambda_{r}\right)$, thus its weight diagram only has $\times$ 's and $\emptyset$ 's.

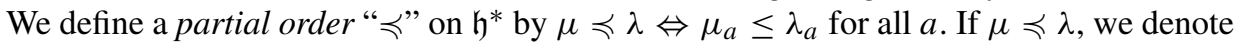
their relative level to be $|\lambda-\mu|=\sum_{a=1}^{m}\left(\lambda_{a}-\mu_{a}\right)$.

For $1 \leq a<b \leq r$, we use $\mathfrak{g}_{[a, b]}$ to denote the subalgebra of $\mathfrak{g}$ generated by the root vectors $X_{\alpha}$ with roots $\alpha$ in $\left\{\epsilon_{p}-\epsilon_{q}, \pm\left(\epsilon_{p}-\delta_{q}\right), \delta_{p}-\delta_{q} \mid a \leq p, q \leq b\right\}$. The weight $\lambda$ restricted to $\mathfrak{g}_{[a, b]}$ is denoted by $\lambda_{[a, b]}$; its diagram is obtained from that of $\lambda$ by deleting the first $a-1$ and the last $r-b+1 \times$ 's.

In the following, we will fix $\lambda$ and use $1,2, \ldots$ to denote paths and their corresponding primitive weights, and $v(\sqrt{1}), \ldots$ to denote the corresponding primitive vectors. If two symbols are put together, e.g., 1 2, it always means a path which is the disjoint sum of two subpaths 1 , 2.

Lemma 5.12. Suppose $i, i=1,2,3,4$, are paths with range $r([1), r(2]) \subset[1, k]$ and $r(3), r(4) \subset[k+1, r]$ for some $k$. If $1, \ldots \rightarrow 2$ and $3 \rightarrow-\ldots \rightarrow$ are chains in the primitive weight graph $P(K(\lambda))$ of $K(\lambda)$, then $13, \cdots \rightarrow 2$ is also a chain in $P(K(\lambda))$. 
Proof. The general result will follow from two special cases: (i) $3=4$, (ii) $1=2$.

(i) Suppose $3=4$. First assume $v(3)$ is strongly primitive. We have $\lambda_{[1, k]}=3_{[1, k]}$ and 1, 2 are paths of $\lambda_{[1, k]}$. Note from (5.7) that any prime coefficient $b_{i}$ of $v(3)$ has the form $\prod_{\alpha \in B^{\prime}} X_{-\alpha}$, where $B^{\prime} \subset\left\{\epsilon_{a}-\delta_{b} \mid k+1 \leq a, b \leq r\right\}$. Thus if we regard $K(\lambda)$ as a $\mathfrak{g}_{[1, k]}$-module, then the $\mathfrak{g}_{[1, k]}$-submodule generated by $v(3)$ is $\mathrm{U}_{\left(\mathfrak{g}_{[1, k]}\right) v(3)}=$ $\mathrm{U}\left(\mathfrak{g}_{[1, k]}^{-}\right) v(3)$, which is in fact the Kac module $K\left(3_{[1, k]}\right)$ by Lemma 5.11 . Therefore

$$
1 \rightarrow 2 \text { in } K(\lambda) \Leftrightarrow 1 \rightarrow 2 \text { in } K\left(\lambda_{[1, k]}\right) \Leftrightarrow 13 \rightarrow 23 \text { in } K(\lambda) .
$$

Thus the result follows in this case.

Next assume $v(3)$ is not strongly primitive. Let 5 be any primitive weight in the space $S:=\mathrm{U}\left(\mathfrak{g}^{+}\right) \mathfrak{g}^{+} v(3)=\mathrm{U}\left(\mathfrak{g}_{+1}\right) \mathfrak{g}_{+1} v(3)$. Then we have a module in which $3 \stackrel{e}{e}$ 5. Dually, we have a module in which 3 ㄴ.. 5, so we have a highest weight module (with highest weight 5) in which $3 \leftarrow \ldots-5$. Thus $3 \in P(K(5))$. In particular $3 \preccurlyeq 5$, such a path 5 must have range within $[k+1, r]$.

We want to prove that we do not have

$$
1][-\ldots-5 \text { in } K(\lambda) .
$$

If we assume (5.11), then we have a highest weight module, denoted by $M$, with highest weight $\mu:=5$ in which $13 \leftarrow \ldots-\mu$. Since $3 \in P(K(\mu)), 3$ corresponds to a path of $\mu$, which we denote by $3^{\prime}$ ( 3 and $3^{\prime}$ are the same weights, but correspond to different paths in different weight diagrams of weights $\lambda$ and $\mu$ ). As the relative level $|\mu-3|<|\lambda-3|$, by induction hypothesis, we may suppose $1\left[3^{\prime} \leftarrow \ldots-3^{\prime} \leftarrow \ldots-\mu\right.$ in $K(\mu)$. Thus in $M$, we

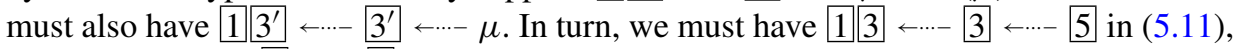
which contradicts $3 \rightarrow \ldots \rightarrow 5$ in $K(\lambda)$.

The above shows that the subquotient $N$ of $K(\lambda)$ given by $N=\mathrm{U}(\mathfrak{g}) v(3) / \mathrm{U}(\mathfrak{g}) S$ is a highest weight module with highest weight 3 , and the $\mathfrak{g}_{[1, k]}$-submodule generated by $v(3])$ in $N$ is the Kac module $K\left(3_{[1, k]}\right)$. Thus again we have (5.10).

(ii) Now suppose $1=2$. In this case, we shall work with $\mathfrak{g}_{0}$-lowest weight vectors instead of $\mathfrak{g}_{0}$-highest weight vectors. As in (i), we only need to consider the case when the $\mathfrak{g}_{0}$-lowest weight vector $\bar{v}(1)$ is strongly primitive. We have $\lambda_{[k+1, r]}=1_{[k+1, r]}$ and 3, 4 are paths of $\lambda_{[k+1, r]}$. Note from (5.9) that any lowest prime coefficient $b_{i}$ of $\bar{v}(1)$ has the form $\prod_{\alpha \in B^{\prime}} X_{-\alpha}$, where $B^{\prime} \subset\left\{\epsilon_{a}-\delta_{b} \mid 1 \leq a, b \leq k\right\}$. Thus if we regard $K(\lambda)$ as a $\mathfrak{g}_{[k+1, r]}$-module, then the $\mathfrak{g}_{[k+1, r]}$-submodule generated by $\bar{v}([1)$ is in fact the Kac module $K\left(1_{[k+1, r]}\right)$. Therefore we have the result as in (i).

Lemma 5.13. Suppose $r(\underline{i}), r\left(\left(^{\prime}\right) \subset\left[a_{i}, b_{i}\right], b_{i}<a_{i+1}, i=1, \ldots, k\right.$. Then

$$
i-\cdots \rightarrow i^{\prime}, i=1, \ldots, k \Leftrightarrow 1 \cdots k \rightarrow 1^{\prime} \cdots k^{\prime} .
$$

Proof. The part " $\Rightarrow$ " can be obtained by Lemma 5.12. Now we prove " $\Leftarrow$ ". We prove $k \rightarrow k^{\prime}$ (the proof of $\bar{i} \rightarrow \ldots \rightarrow \bar{i}^{\prime}$ for $i<k$ is similar). By Lemma 5.12, we have

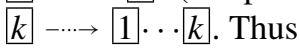

$$
k \rightarrow[]^{\prime} \cdots k^{\prime} .
$$


If $\mu:=k$ is a path without bridges, then it generates a highest weight module $M$, and so $\left[1^{\prime} \cdots \mid k\right]^{\prime} \preccurlyeq \mu$. This forces $\left[k^{\prime} \preccurlyeq \mu\right.$, and $\left[1^{\prime} \cdots|k|^{\prime}\right.$ corresponds to a path of $\mu$, which must have the form $1^{\prime} \cdots a^{\prime} k^{\prime \prime}$, where $a=k-1$ and $k^{\prime \prime}$ is a path of $\mu$ such that $k{ }^{\prime}=k^{\prime \prime}$ as weights. Note that in the Kac module $K(\mu)$, we have

$$
\mu \rightarrow \cdots \rightarrow k^{\prime \prime}-\cdots \rightarrow 1^{\prime} \cdots a^{\prime} k^{\prime \prime} .
$$

Thus in every highest weight module with highest weight $\mu$ in which (5.12) holds, (5.13) must also hold. Therefore in $M$, we have (5.13). In particular $k \rightarrow k^{\prime}$ in $K(\lambda)$.

Now suppose $k$ is a bridge. Let $k^{\prime \prime}$ be any path of $\lambda$ such that $k \stackrel{e}{-\cdots \rightarrow} k^{\prime \prime}$. If $k^{\prime \prime}-\ldots \rightarrow k^{\prime}$, then we have the result. Otherwise, we take $M$ to be the highest weight module which is the subquotient of $K(\lambda)$ given by the submodule generated by $v(k)$ modulo that generated by all $k{ }^{\prime \prime}$. Then using arguments as in the previous paragraph, we obtain the result.

Lemma 5.14. Suppose $\mu=1, v=2$ are two paths without bridges. Then

$$
1-\cdots \rightarrow 2 \Leftrightarrow 1 \text { is a subpath of } 2 \text {. }
$$

Proof. Suppose $1-\cdots \rightarrow 2$. Since 1 is strongly primitive in $K(\lambda)$ (cf. Remark 5.7(2)), we obtain $v \in P(K(\mu))$. Thus we have $\mu=L_{\theta}(v)$ and $\lambda=R_{\Theta}(v)$ (cf. Definition 5.1). Similar to the definition of blocks of a left path, we can divide the right path $R_{\Theta}(v)$ into the disjoint sum of its indecomposable blocks, say $R_{\Theta}(v)=1 \cdots k$ (here we use $i$ 's to denote right paths). If $R_{\theta}(v)$ is not a subpath of $R_{\Theta}(v)$, then $R_{\theta}(v)$ contains at least a right move $R_{i}(v)=R_{i j}(v)$ which does not appear in $R_{\Theta}(v)$. By Remark 5.7(3), we may suppose that $R_{i}(v)$ is a right move which is after $a$ but before $b$, where $b=a+1$ for some $1 \leq a \leq k$. Then one sees that the $j$-th entry of $\mu=R_{\theta}(v)$ is larger than that of $\lambda=R_{\Theta}(\nu)$. Thus $\mu \nprec \lambda$, contradicting $\mu \in P(K(\lambda))$. Thus $R_{\theta}(\nu)$ is a right subpath of $R_{\Theta}(v)$. It then follows that 1 is a (left) subpath of 2 .

Next suppose 1 is a subpath of 2 . Dividing 2 into the disjoint sum of its blocks and dividing 1 into a disjoint sum of subpaths accordingly, then using Lemma 5.13, we can suppose that 2 is indecomposable. So suppose $r(2)=[a, b]$ and $\ell(2])=b+1-a$ (cf. Remark 5.7(3)). Thus we can regard 2 as the path for $\mathfrak{g}_{[1, b]}$, i.e., we can suppose $r=b$. By considering $\mathfrak{g}_{0}$-lowest weight vectors (as in part (ii) of the proof of Lemma 5.12 ), and observing that each $\mathfrak{g}_{0}$-lowest weight vector of a path $i$ with range in $[a, b]$ is the same as that of the $\left(\mathfrak{g}_{[a, b]}\right)_{0}$-lowest weight vector of $i$ regarded as a path for the Lie superalgebra $\mathfrak{g}_{[a, b]}$, we can regard 1, 2] as paths for $\mathfrak{g}_{[a, b]}$, i.e., we can suppose $a=1, b=r$. But in this case 2 is the bottom path (cf. Remark 5.7(1)). Therefore $1 \rightarrow 2$.

Remark 5.15. It follows from Lemma 5.14 that we have the following exact chain of length $r=\sharp(\lambda)$ for the Kac module $K(\lambda)$ :

$$
L_{\emptyset} \rightarrow L_{[1,1]} \rightarrow L_{[1,2]} \rightarrow \cdots \rightarrow L_{[1, r]},
$$

where the paths $L_{[1, k]}$ are defined in Remark 5.7(2). In particular, $L_{\emptyset}$ corresponds to $K(\lambda)$ itself and $L_{[1, r]}$ to the bottom composition factor $\bar{L}(\lambda)$. The exactness of the chain is deduced from Corollary 4.5. Note that (5.14) is nothing other than the chain (4.2). 
The following result can be easily proven by using Theorems 3.6 and 3.2.

Lemma 5.16. For every path $P$ with length $k$, there exists an exact chain of length $r=$ $\sharp(\lambda)$ in $P(K(\lambda))$ of the form

$$
L_{\emptyset}=0 \rightarrow 1 \rightarrow \cdots \rightarrow r=L_{[1, r]}, \quad \text { with } k=P .
$$

Proof. Obviously the chain $L_{\emptyset} \rightarrow \cdots \rightarrow P \rightarrow L_{[1, r]}$ is in $P(K(\lambda))$. We can always insert vertices in the intervals $\left[L_{\emptyset}, P\right]$ and $\left[P, L_{[1, r]}\right]$ to turn it into an exact chain, the length of which will be denoted by $l$. Theorem 3.2 and Corollary 4.5 require $l \leq r$. If $l<r$, the socle and radical filtrations of $K(\lambda)$ would not coincide, contradicting the fact that the Jantzen filtration for $K(\lambda)$ is the unique Loewy filtration (Theorem 3.6). Hence the resulting exact chain must be of the form (5.15) with $k=P$ for some $k$, where the submodule corresponding to $i$ belongs to the $i$-th layer of the Jantzen filtration.

Lemma 5.17. A path with bridges is not strongly primitive.

Proof. Let 1 be a path with bridges. By Lemma 5.13, we can assume 1 is indecomposable. Furthermore as in the proof of Lemma 5.14, we can suppose $r(1)=[1, r]$. In this case, 1 must contain the left path $L_{1 r}$. One can easily see that the bottom path $L_{B}$ (cf. Remark 5.7(2)), whose primitive vector can be generated by that of 1 , is not $\preccurlyeq 1$. Therefore, 1 cannot be strongly primitive.

\subsection{Primitive weight graphs of Kac modules}

The following theorem completely determines the primitive weight graph of the Kac module $K(\lambda)$.

Theorem 5.18. For any two paths 1 and 2 , we have $1 \rightarrow 2$ if and only if $\ell(1)=$ $\ell(2])-1$, and one of the following holds.

(1) 1 is a subpath of 2];

(2) 1 is a bridge, and 2 is obtained from 1 by replacing some left move $L_{i j}$ appearing in 1 with $i<j$ by two moves $L_{i a}, L_{b j}$ for some $i \leq a \leq j$ and $b$ satisfying $a \leq b \leq j$ being the smallest such that the result is a path.

Proof. Suppose $\ell(1)=\ell(2)-1$. First assume 1 is a subpath of 2 . We can suppose 2 is indecomposable by Lemma 5.13. Suppose $r(2])=[a, b]$. Then $r(1) \subset[a, b]$. As in the proof of Lemma 5.14, we can regard 1, 2 as paths for $\mathfrak{g}_{[a, b]}$. Thus without losing generality, we can suppose $a=1, b=r$. Being indecomposable, 2 must contain the left move $L_{1 r}$. We denote $v=1, \mu=2$.

Consider the dual Kac module $K(\lambda)^{*}$, which is the Kac module $K\left(\lambda^{\#}\right)$ with $\lambda^{\#}=$ $2 \rho_{1}-\bar{\lambda}$ (recall (5.8) for notation $\bar{\lambda}$ ) by noting that the lowest weight in $K(\lambda)$ is $\overline{\lambda-2 \rho_{1}}=$ $\bar{\lambda}-2 \rho_{1}$. Note that the dual module of any $L_{\mu}$ is the module $L_{\mu^{*}}$ with $\mu^{*}=2 \rho_{1}-\overline{R_{\Theta}(\mu)}$, where $\Theta=(1, \ldots, 1)$. Since 2 contains the left move $L_{1, r}$, we see that when we write $\lambda$ in terms of right paths of $\mu$, we must have $\lambda=R_{\theta}(\mu)$ for some $\theta \in\{0,1\}^{r}$ with $\theta_{1}=1$, and we find that the first entries of $\lambda$ and $R_{\Theta}(\mu)$ are the same. This shows that the $r$-th 
entries of $\lambda^{\#}$ and $\mu^{*}$ are the same. This implies that when we write $\mu^{*}$ as a left path of $\lambda^{\#}$, which we denoted by 2$]^{*}$, we must have $\left.r(2)^{*}\right) \subset[2, r]$. In particular $2^{*}$ has depth $<r=d(2)$. Thus by induction on the depth of the path, we can assume $v^{*} \rightarrow \mu^{*}$ in $K(\lambda)^{*}$. Thus $1 \rightarrow 2$ in $K(\lambda)$.

Next suppose case (2) of Theorem 5.18 occurs. In this case we can suppose 1 is indecomposable and $r(1)=[1, r]$. Then we can prove $1 \rightarrow 2$ in a similar way to the above.

Now suppose $1 \rightarrow 2$. By Lemma 5.16, we must have $\ell(2) \geq \ell(1)+1$. Denote $v=1, \mu=2$. Then

$$
\text { either } v \rightarrow \mu \text { or } \quad v \leftarrow \mu \text { is a highest weight module. }
$$

In the former case, $\mu \in P(K(v))$. So $\mu$ corresponds to a path of $v$. Denote this path by 2 '; then we must have $\ell\left(2{ }^{\prime}\right)=1$. Otherwise we do not have $v \rightarrow \mu$ in the Kac module $K(v)$ by Lemma 5.14, and the first case of (5.16) cannot happen. Suppose $2{ }^{\prime}=L_{a b}$ for some $a, b$. Then as paths of $\lambda$, we must have $2=1 L_{a b}$ (i.e., 2 is obtained from 1 by adding one more move $L_{a b}$ ), as otherwise $\mu$ cannot be a primitive weight of $\lambda$. Thus $\ell(1])=\ell(\sqrt{2})-1$ and 1 is a subpath of 2 .

In the second case of (5.16), we have

$$
\nu \stackrel{e}{\rightarrow} \mu \quad \text { in } K(\lambda) .
$$

Thus $v \in P(K(\mu))$. If $\ell(1])<\ell(2])-1$, then in the Kac module $K(\mu)$, there exists some $\tau \in P(K(\mu))$ such that $v \leftarrow \ldots-\tau \leftarrow \cdots-\mu$. In turn, we must have $v-\ldots \rightarrow \tau-\ldots \rightarrow \mu$ in $K(\lambda)$, a contradiction with (5.17). Thus $\ell(1)=\ell(2)-1$. From Lemma 5.13, we can then assume that 1 is indecomposable. Thus as above, we can suppose $r(1)=[1, r]$. Now using similar arguments to those in the second paragraph of this proof, by induction on the depth of the path, we can prove that 2 is obtained from 1 by case (2) of the theorem.

Remark 5.19. Part (2) of Theorem 5.18 can also be expressed in terms of permissible codes or boundary strip removals of the composite Young diagram of $\lambda[18,33]$.

Acknowledgments. We thank Catharina Stroppel for correspondence. This work was supported by the Australian Research Council and National Science Foundation of China (grant no. 10825101).

\section{References}

[1] Andersen, H. H.: Filtrations of cohomology modules for Chevalley groups. Ann. Sci. École Norm. Sup. (4) 16, 495-528 (1983) Zbl 0545.20030 MR 0740588

[2] Andersen, H. H.: Jantzen's filtrations of Weyl modules. Math. Z. 194, 127-142 (1987) Zbl 0595.20038 MR 0871225

[3] Beilinson, A. A., Bernstein, J.: Localisation de $g$-modules. C. R. Acad. Sci. Paris Sér. I Math. 292, 15-18 (1981) Zbl 0476.14019 MR 0610137

[4] Beilinson, A. A., Bernstein, J.: A proof of Jantzen conjectures. In: I. M. Gelfand Seminar, Adv. Soviet Math. 16, Part 1, Amer. Math. Soc., Providence, RI, 1-50 (1993) Zbl 0790.22007 MR 1237825 
[5] Beilinson, A. A., Ginzburg, V., Soergel, W.: Koszul duality patterns in representation theory. J. Amer. Math. Soc. 9, 473-527 (1996) Zbl 0864.17006 MR 1322847

[6] Boe, B. D., Collingwood, D. H.: Multiplicity free categories of highest weight representations. I, II. Comm. Algebra 18, 947-1032, 1033-1070 (1990) Zbl 0757.17005 MR 1059940

[7] Brown, K. A., Goodearl, K. R.: Lectures on Algebraic Quantum Groups. Birkhäuser (2002) Zbl 1027.17010 MR 1898492

[8] Brundan, J.: Kazhdan-Lusztig polynomials and character formulae for the Lie superalgebra $\mathfrak{g}(m \mid n)$. J. Amer. Math. Soc 16, 185-231 (2002) Zbl 1050.17004 MR 1937204

[9] Brundan, J.: Stroppel, C.: Highest weight categories arising from Khovanov's diagram algebra I, II, III, IV. Moscow Math. J. 11, 685-722 (2011); Transform. Groups 15, 1-45 (2010); Represent. Theory 15, 170-243 (2011); J. Eur. Math. Soc. 14, 373-419 (2012) Zbl 1205.17010(II) MR 2600694(II) MR 2781018(III)

[10] Brylinski, J.-L., Kashiwara, M.: Kazhdan-Lusztig conjecture and holonomic systems. Invent. Math. 64, 387-410 (1981) Zbl 0473.22009 MR 0632980

[11] Cheng, S. J., Lam, N.: Irreducible characters of general linear superalgebra and super duality. Comm. Math. Phys. 298, 645-672 (2010) Zbl 1217.17004 MR 2670923

[12] Cheng, S. J., Lam, N., Wang, W.: Super duality and irreducible characters of ortho-symplectic Lie superalgebras. Invent. Math. 183, 189-224 (2011) Zbl pre05852689 MR 2755062

[13] Cheng, S. J., Wang, W., Zhang, R. B.: Super duality and Kazhdan-Lusztig polynomials. Trans. Amer. Math. Soc. 360, 5883-5924 (2008) Zbl pre05358271 MR 2425696

[14] Cheng, S. J., Zhang, R. B.: Analogue of Kostant's u-cohomology formula for the general linear superalgebra. Int. Math. Res. Notices 2004, no. 1, 31-53 Zbl 1096.17005 MR 2036954

[15] Collingwood, D. H., Irving, R. S., Shelton, B.: Filtrations on generalized Verma modules for Hermitian symmetric pairs. J. Reine Angew. Math. 383, 54-86 (1988) Zbl 0631.22014 MR 0921987

[16] Gabber, O., Joseph, A.: Towards the Kazhdan-Lusztig conjecture. Ann. Sci. École Norm. Sup. (4) 14, 261-302 (1981) Zbl 0476.17005 MR 0644519

[17] Gruson, C., Serganova, V.: Cohomology of generalized supergrassmannians and character formulae for basic classical Lie superalgebras. Proc. London Math. Soc. 101, 852-892 (2010) Zbl 1216.17005 MR 2734963

[18] Hughes, J. W. B., King, R. C., van der Jeugt, J.: On the composition factors of Kac modules for the Lie superalgebras sl $(m \mid n)$. J. Math. Phys. 33, 470-491 (1992) Zbl 0747.17003 MR 1145343

[19] Hughes, J. W. B., King, R. C., van der Jeugt, J.: private communication

[20] Humphreys, J. E.: Representations of Semisimple Lie Algebras in the BGG Category O O. Grad. Stud. Math. 94, Amer. Math. Soc., Providence, RI (2008) Zbl 1177.17001 MR 2428237

[21] Irving, R. S.: A filtered category $\mathcal{O}_{S}$ and applications. Mem. Amer. Math. Soc. 83, no. 419, vi+117 pp. (1990) Zbl 0715.22017 MR 0978603

[22] Jantzen, J. C.: Kontravariante Formen auf induzierten Darstellungen halbeinfacher LieAlgebren. Math. Ann. 226, 53-65 (1977) Zbl 0372.17003 MR 0439902

[23] Jantzen, J. C.: Moduln mit einem höchsten Gewicht. Lecture Notes in Math. 750, Springer, Berlin (1979) Zbl 0426.17001 MR 0552943

[24] Kac, V. G.: Lie superalgebras. Adv. Math. 26, 8-96 (1977) Zbl 0366.17012 MR 0486011

[25] Kazhdan, D.: Lusztig, G.: Representations of Coxeter groups and Hecke algebras. Invent. Math. 53, 165-184 (1979) Zbl 0499.20035 MR 0560412

[26] Musson, I., Serganova, V.: Combinatorics of character formulas for the Lie superalgebra $\mathfrak{g l}(m, n)$. Transform. Groups 16, 555-578 (2011) Zbl pre05982615 MR 2806501

[27] Scheunert, M.: The Theory of Lie Superalgebras. An Introduction. Lecture Notes in Math. 716, Springer, Berlin (1979) Zbl 0407.17001 MR 0537441 
[28] Serganova, V.: Kazhdan-Lusztig polynomials and character formula for the Lie superalgebra gl $(m \mid n)$. Selecta Math. 2, 607-654 (1996) Zbl 0881.17005 MR 1443186

[29] Serganova, V.: Characters of irreducible representations of simple Lie superalgebras. In: Proc. Int. Congress Math. Berlin (1998), Vol. II, Doc. Math. 1988, Extra Vol. II, 583-593 Zbl 0898.17002 MR 1648107

[30] Soergel, W.: Andersen filtration and hard Lefschetz. Geom. Funct. Anal. 17, 2066-2089 (2008) Zbl 1143.22012 MR 2399092

[31] Stroppel, C.: Parabolic category $\mathcal{O}$, perverse sheaves on Grassmannians, Springer fibres and Khovanov homology. Compos. Math. 145, 954-992 (2009) Zbl 1187.17004 MR 2521250

[32] Stroppel, C., Webster, B.: 2-block Springer fibers: convolution algebras and coherent sheaves. Comment. Math. Helv. 87, 477-520 (2012)

[33] Su, Y.: Composition factors of Kac modules for the general linear Lie superalgebras. Math. Z. 252, 731-754 (2006) Zbl 1106.17008 MR 2206623

[34] Su, Y., Hughes, J. W. B., King, R. C.: Primitive vectors of Kac-modules of the Lie superalgebra sl $(m \mid n)$. J. Math. Phys. 41, 5064-5087 (2000) Zbl 0988.17004 MR 1765833

[35] Su, Y., Zhang, R. B.: Cohomology of Lie superalgebras $\mathfrak{s l}_{m \mid n}$ and $\mathfrak{o s p}_{2 \mid 2 n}$. Proc. London Math. Soc. 94, 91-136 (2007) Zbl 1118.17005 MR 2293466

[36] Su, Y., Zhang, R. B.: Character and dimension formulae for general linear superalgebra. Adv. Math. 211, 1-33 (2007) Zbl 166.17002 MR 2313526

[37] Su, Y., Zhang, R. B.: Generalised Verma modules for the orthosymplectic Lie superalgebra $\mathfrak{o s p}_{k \mid 2}$. J. Algebra 357, 94-115 (2012)

[38] van der Jeugt, J., Hughes, J. W. B., King, R. C., Thierry-Mieg, J.: A character formula for singly atypical modules of the Lie superalgebra $\mathrm{sl}(m / n)$. Comm. Algebra 19 (1991), 199222. Zbl 0721.17004 MR 1092559

[39] van der Jeugt, J., Zhang, R. B.: Characters and composition factor multiplicities for the Lie superalgebra gl $(m / n)$. Lett. Math. Phys. 47, 49-61 (1999) Zbl 0932.17009 MR 1669394

[40] Zou, Y. M.: Categories of finite-dimensional weight modules over type I classical Lie superalgebras. J. Algebra 180, 459-482 (1996) Zbl 0843.17001 MR 1378540 